MPP-2005-83

August 8, 2005

\title{
Regular solutions to higher order curvature Einstein-Yang-Mills systems in higher dimensions
}

\author{
Peter Breitenlohner, ${ }^{\ddagger}$ Dieter Maison ${ }^{\ddagger}$ and D. H. Tchrakian ${ }^{\dagger \star}$ \\ $\ddagger_{\text {Max-Planck-Institut für Physik }}$ \\ Werner-Heisenberg-Institut \\ Föhringer Ring 6, D-80805 München, Germany \\ ${ }^{\dagger}$ Department of Mathematical Physics, National University of Ireland Maynooth, \\ Maynooth, Ireland \\ ${ }^{\star}$ School of Theoretical Physics - DIAS, 10 Burlington Road, Dublin 4, Ireland
}

\begin{abstract}
We study regular, static, spherically symmetric solutions of Yang-Mills theories employing higher order invariants of the field strength coupled to gravity in $d$ dimensions. We consider models with only two such invariants characterised by integers $p$ and $q$. These models depend on one dimensionless parameter $\alpha$ leading to one-parameter families of regular solutions, obtainable by numerical solution of the corresponding boundary value problem. Much emphasis is put on an analytical understanding of the numerical results.
\end{abstract}




\section{Introduction}

Regular, static, and finite energy solutions to the Einstein-Yang-Mills (EYM) equations have presented a subject of interest since the work of Bartnik and McKinnon [1] (BM) in 4 spacetime dimensions. Besides the regular solutions also corresponding black hole solutions were found [2, 3, 4, representing novel examples of hairy black holes. In addition, these classical solutions are of interest also as the 'solitons' of the low energy effective actions of superstring theory [5], since both gravitational and non Abelian gauge fields occur in the latter and in gauged supergravities. More specifically, such solutions (especially the black holes) have an important role to play in quantum gravity [6], and also on (higher dimensional) $D$-branes [7. In this wider context therefore, the construction of such solutions in spacetimes of dimensionality higher than $d=4$ is of actual interest. This is the purpose of the present work, namely the study of EYM systems in arbitrary spacetime dimensions $d$.

Although the YM theory in flat space has no 'soliton' type solutions due to its scaling properties, the coupling to gravity changes the picture. Bartnik and McKinnon [1] discovered a discrete family of static, spherically symmetric globally regular solutions. However, the scaling properties of the EYM theory expressed by a 'Virial Theorem' forbid such solutions for $d \geq 5$; while the scaling dimension of static gravitational fields is $d-3$ the one of the YM fields is equal to $d-5$. The virial theorem implies that non-trivial regular solutions require terms in the action with scaling dimensions of different sign. The way out proposed in [8, 9] is to use higher order invariants for gravity and/or YM fields. The choice of models boils down, in principle, to the selection of various terms in the gravitational and non Abelian hierarchies of increasing orders in the respective curvatures, namely the Riemann and the YM curvatures, which are reparametrisation and gauge invariant. There is an important restriction, namely that one considers only those Lagrange densities that are constructed from antisymmetrised $2 p$ curvature forms, and exclude all other powers of both Riemann and YM curvature 2-forms. (In the gravitational case this results in the familiar Gauss-Bonnet Lagrangean.) As a result, only velocity-squared fields appear in the Lagrangean, which is what is needed for physical reasons. Here we will add only the minimal number of such higher order terms that are necessitated by the requirements of the virial theorem. This criterion makes the inclusion of higher order gravitational terms unnecessary and in fact the inclusion of higher order gravitational terms does not seem to alter the qualitative properties of the classical solutions [8]. From the viewpoint of classical solutions, indeed, such terms seem redundant. They become, however, important in the mathematically interesting situations where the first member of the YM hierarchy, namely the usual YM term, is absent. The situation concerning higher order YM curvature terms in the string theory effective action is complex and as yet not fully resolved. While YM terms up to $F^{4}$ arise from (the non Abelian version of) the Born-Infeld action [10, it 
appears that this approach does not yield all the $F^{6}$ terms [11. Terms of order $F^{6}$ and higher can also be obtained by employing the constraints of (maximal) supersymmetry [12]. The results of the various approaches are not identical.

Thus we end up adding only higher order YM terms to the usual EYM Lagrangean. The YM hierarchy employed is

$$
\mathcal{L}_{P}=\sum_{p=1}^{P} \frac{\tau_{p}}{2(2 p) !} \operatorname{Tr} F(2 p)^{2},
$$

$F(2 p)$ denoting the $p$ fold totally antisymmetrised products

$$
F(2 p) \equiv F_{\mu_{1} \mu_{2} \ldots \mu_{2 p}}=F \wedge F \wedge \ldots \wedge F, \quad p \text { times }
$$

of the YM curvature, $F(2)=F_{\mu \nu}$, in this notation. Clearly, the highest value $P$ of $p$ in Eq. (11) is finite and depends on the dimensionality $d$ of the spacetime. To complete the definition of the models (11) the gauge group $G$ must be specified. With our aim in the present paper, of constructing static spherically symmetric solutions in $d$ spacetime dimensions, the smallest possible such gauge group is $G=S O(d-1)$ requiring $d>4$.

In [8, 9] the simplest possibility $P=2$ was chosen. The scaling dimension of the $p=2$ term is equal to $d-9$, yielding a negative contribution in the virial theorem for $d<9$. As a consequence these gravitating YM models in spacetime dimensions $d=5,6,7$, and 8 were found to support particle like solutions ${ }^{1}$. Here we will consider more generally models involving two arbitrary terms of the sum in Eq. (11), say $F(2 p)$ and $F(2 q)$. For simplicity let us call them $p$-q-models. Like the 1-2-model these simplest nontrivial models possess one dimensionless parameter $\alpha$ providing a measure of the strength of the gravitational self-interaction of the YM configurations.

The regular solutions reported in [8, 9, which were constructed numerically, featured numerous novel and exotic properties, some of which were not understood or explained at a more fundamental level. The purpose of the present work is specifically to understand these properties analytically, and then substantiate the conclusions and predictions numerically. In fact, our analysis immediately generalises to the general $p$ - $q$-models, all showing the same basic structure. In this context it also turns out to be useful to interpolate in the dimension, i.e. take the dimension parameter $d$ to be an arbitrary real number. This is possible since the reduced one dimensional equations of motion for static, spherically symmetric configurations depend parametrically on $d$.

The properties of the solutions alluded to in [8, 9] are:

1. The solutions form one parameter families parametrised by $\alpha$. It was found that $\alpha$ increases from the flat limit zero up to some $\alpha_{\max }$ and then turns back.

\footnotetext{
${ }^{1}$ Taking only the $p=2$ term one finds generalisations of the BM solutions in these dimensions
} 
2. In $d=6,7$, and 8 , where there is a finite-energy gravity-decoupling solution, two solutions appear to exist for most values of $\alpha$, up to $\alpha_{\max }$. Starting from the flat $F(2)+F(4)$ solution $\alpha$ increases from zero to a maximal value $\alpha_{\max }$ and then runs back to zero for $d=8$, while for $d=6$ and 7 the families end at some finite non-zero value of $\alpha$

3. In $d=5$ there is no flat $F(2)+F(4)$ solution, but the relevant flat solution is the instanton of the pure $F(2)$ theory considered as a static solution of the $d=5$ theory. After a suitable rescaling both gravity and the $F(4)$ term decouple in the limit $\alpha \rightarrow 0$. Again $\alpha$ turns back after reaching some maximal value, but then seems to converge to some limiting critical value $\alpha_{c}$ after performing a finite or infinite number of oscillations.

4. Unlike in the case of $d=4$ EYM theory no multi-node solutions were found.

In the present paper we will try to clarify the findings desribed above with particular emphasis on the critical values of the parameter $\alpha$ marking the endpoint of the one-parameter families of solutions. Based on experience with other systems like self-gravitating magnetic monopoles [13] we propose that the critical solutions run into certain fixed points (f.p.) of the dynamical system represented by the reduced field equations, a system of nonlinear ordinary differential equations. Employing the standard method of linearisation at the f.p. it is possible to study the analytical properties of solutions coming close to the f.p. and derive asymptotic expressions for their parameters. Constructing solutions starting at the f.p. itself provides a direct method to determine critical parameters like $\alpha_{c}$ yielding much more precise values than extrapolation from solutions coming only close to it. In fact, our analysis leads to a rather clear picture of the results listed above.

Concerning point $2 .,(d=6,7$, and 8$)$ we claim that the upper branch of the solution family runs back all the way to $\alpha=0$, the limit (after suitable rescaling decoupling the $F(2)$ part) being the analogue of the BM solution for the $F(4)$ model. As $\alpha$ tends back to zero the solutions for $d=6$ and $d=7$ come very close to a fixed point already known from the EYM theory in $d=4$ related to the behaviour at the horizon of the extremal Reissner-Nordstrøm black hole [14, 13. Our analytical analysis, however, excludes a bifurcation with solutions running into the fixed point. Although our improved numerical computations support this analytical result for $d=7$, for $d=6$ the approach to the f.p. is too close to resolve this question numerically.

The limiting solution for $d=5$ described in 3 . is related to a new type of fixed point described in Section 3. The resulting structure is comparable to the limit of infinite Higgs mass for magnetic monopoles [13].

As to 4 . we show that multi-node solutions exist, but only for $d$ between 4 and 5 , i.e. not for the integer dimensions studied in [8, 9]. 
In addition to clarifying the points $1 .-4$. above, we have carried our conclusions further, to verify that these qualitative features repeat themselves for the general $p$ - $q$-models. Particularly the models with $q=p+1$ show a certain degree of periodicity modulo $4 p+1$.

\section{The reduced one dimensional theory}

The Lagrangean of the Yang-Mills hierarchy in $d$ spacetime dimensions introduced in 8 including the generic $p$-th member, is

$$
\mathcal{L}_{Y M}=\sum_{p} \frac{\tau_{p}}{2 \cdot(2 p) !} \operatorname{tr} F(2 p)^{2},
$$

where the $2 p$ form $F(2 p)$ is the totally antisymmetrised product of the YM field strength $F(2)$. For the metric the static spherically symmetric ansatz with $d-1$ space-like dimensions is

$$
d s^{2}=d s_{2}^{2}-r^{2} d \Omega_{d-2}^{2},
$$

where $d s_{2}^{2}$ is the metric on the two dimensional orbit space factorising out the action of the rotation group and $d \Omega_{d-2}^{2}$ the invariant line element of $S^{d-2}$. The $2 \mathrm{~d}$ metric can always be brought to the diagonal form

$$
d s_{2}^{2}=e^{2 \nu} d t^{2}-e^{2 \lambda} d R^{2} .
$$

Considering only time independent solutions we naturally take $t$ to be the Killing time, while the radial coordinate $R$ remains arbitrary. Plugging this ansatz into the standard Einstein-Hilbert action results in the reduced one dimensional Lagrangean

$$
L_{G}=-\frac{1}{2 G} \mathrm{e}^{\nu+\lambda} r^{d-4}\left[d-3+\mathrm{e}^{-2 \lambda}\left(\nu^{\prime}\left(r^{2}\right)^{\prime}+(d-3)\left(r^{\prime}\right)^{2}\right)\right],
$$

where $G$ denotes Newton's constant. The static spherically symmetric Ansatz for the $S O_{ \pm}(d)$ resp. $S O_{ \pm}(d-1)$ in even resp. odd spacetime dimensional YM field is

$$
A_{0}=0, \quad A_{i}=\left(\frac{1-W}{r}\right) \Sigma_{i j}^{( \pm)} \hat{x}_{j}, \quad \Sigma_{i j}^{( \pm)}=-\frac{1}{4}\left(\frac{1 \pm \Gamma_{c h}}{2}\right)\left[\Gamma_{i}, \Gamma_{j}\right],
$$

where $\Gamma_{c h}$ is the chiral matrix in the appropriate dimensions.

The resulting reduced one-dimensional Lagrangean is

$$
L_{\mathrm{YM}}=\sum_{p} r^{d-4} V^{p-1}\left[a_{p} \mathrm{e}^{\nu-\lambda}\left(W^{\prime}\right)^{2}+b_{p} \mathrm{e}^{\nu+\lambda} r^{2} V\right],
$$

with $V=\left(W^{2}-1\right)^{2} / r^{4}$ and coefficients

$$
a_{p}=\tau_{p} \frac{(d-2) !}{2(2 p-1) !(d-2 p-1) !}=\frac{1}{2} \hat{\tau_{p}}, \quad \text { and } \quad b_{p}=\frac{d-2 p-1}{2 p} a_{p} .
$$


From the form of these cofficients one can see that if $d$ is an integer it must be larger than $2 p$ for the term with $F(2 p)$ to contribute. Thus e.g. for $d=4$ only the $F(2)$ term is present. Therefore we will restrict our analysis to values of $d>2 P$, where $P$ is the maximal value of $p$ in the sum.

An infinitesimal scale transformation of the action $S=\int d R\left(L_{G}+L_{Y M}\right)$ yields a virial theorem. While the gravitational part picks up a factor $d-3$ the $F(2 p)$ terms get a factor $d-4 p-1$. As a consequence non-trivial flat solutions exist only, if the conditions $d-4 P-1<0$ and simultaneously $d-4 p-1>0$ for the first non-vanishing term in the YM part are fulfilled. On the other hand, if $d-4 p-1<0$ for the first non-vanishing term then the gravitational part is required to obtain non-trivial solutions.

Varying the action with respect to the variables $\nu, r$ and $W$ yields three second order ordinary differential equations. Introducing independent variables $N, \kappa$ and $U$ for the first derivatives

$$
N \equiv e^{-\lambda} r^{\prime}, \quad \kappa \equiv r e^{-\lambda} \nu^{\prime}+N, \quad U \equiv e^{-\lambda} W^{\prime}
$$

the equations of motion become

$$
\begin{aligned}
r e^{-\lambda} N^{\prime}= & (\kappa-N) N-2 G \sum_{p} a_{p} V^{p-1} U^{2} \\
r e^{-\lambda} \kappa^{\prime}= & -\frac{1}{2}(d-3)(d-6)-\kappa^{2}+\frac{1}{2}(d-4)(d-5) N^{2} \\
& -G \sum_{p} V^{p-1}\left(a_{p}(d-4 p-2) U^{2}-b_{p}(d+4 p-8) T^{2}\right), \\
r e^{-\lambda} U^{\prime}= & \frac{1}{\sum_{p} a_{p} V^{p-1}}\left[\sum_{p} V^{p-1}((4 p+1-d) N-\kappa) a_{p} U\right. \\
& \left.+2 W T \sum_{p} V^{p-1}\left(p b_{p}-(p-1) a_{p} \frac{U^{2}}{T^{2}}\right)\right]
\end{aligned}
$$

where we have introduced the shorthand $T=\left(W^{2}-1\right) / r$. The variation with respect to $\lambda$ yields the algebraic constraint for the first derivatives

$$
\kappa N-\frac{1}{2}(d-3)+\frac{1}{2}(d-5) N^{2}-G \sum_{p} V^{p-1}\left(a_{p} U^{2}-b_{p} T^{2}\right)=0 .
$$

Subsequently we shall restrict ourselves to models with only two terms $(p 1, p 2)$ in the sum over $p$ denoting them simply as $p$ and $q$. The simplest choice is $p=1$ and $q=2$, the choice made in [8, 9. Suitably rescaling $r$ and the total action the model depends only on the dimensionless parameter $\alpha^{4(q-p)}=G^{2(q-p)}{\hat{\tau_{p}}}^{2 q-1}{\hat{\tau_{q}}}^{{ }^{1-2 p}}$. Taking more terms in the sum does not seem to change the overall picture, but makes the numerical as well as the analytical analysis much more cumbersome. 


\section{The $F(2 p)$ model}

Before we turn to the $F(2 p)+F(2 q)$ model we consider the model with only one $F(2 p)$ term, generalising the system studied by Bartnik and McKinnon [1]. In this case the parameters $G$ and $\hat{\tau}_{p}$ can be completely absorbed by a rescaling of $r$ and the total action. We still have the freedom of choosing a suitable radial

coordinate. As in [14] we impose the condition $e^{\lambda}=r$, call the corresponding radial coordinate $\tau$ and denote $\tau$ derivatives by a dot. Thus we obtain from Eqs. (111)

$$
\begin{aligned}
\dot{r}= & r N, \\
\dot{W}= & r U, \\
\dot{N}= & (\kappa-N) N-V^{p-1} U^{2}, \\
\dot{\kappa}= & 1-\kappa^{2}+\frac{(d-4)(d-5)}{2}\left(N^{2}-1\right) \\
& +\frac{V^{p-1}}{2}\left((4 p+2-d) U^{2}+\frac{(d-2 p-1)(d+4 p-8)}{2 p} T^{2}\right), \\
\dot{U}= & ((4 p+1-d) N-\kappa) U+W T\left(d-2 p-1-2(p-1) \frac{U^{2}}{T^{2}}\right),
\end{aligned}
$$

and the constraints

$$
\begin{aligned}
& W^{2}-1-r T=0, \\
& 2 \kappa N+3-d+(d-5) N^{2}-V^{p-1}\left(U^{2}-\frac{d-2 p-1}{2 p} T^{2}\right)=0 .
\end{aligned}
$$

It turns out to be convenient to introduce new variables $y=V^{p-1} T^{2}$ and $z=U / T$ and rewrite the equations as

$$
\begin{aligned}
\dot{r}= & r N, \\
\dot{W}= & r T z, \\
\dot{T}= & (2 W z-N) T, \\
\dot{N}= & (\kappa-N) N-y z^{2}, \\
\dot{\kappa}= & 1-\kappa^{2}+\frac{(d-4)(d-5)}{2}\left(N^{2}-1\right) \\
& +\left((4 p+2-d) z^{2}+\frac{(d-2 p-1)(d+4 p-8)}{2 p}\right) \frac{y}{2}, \\
\dot{y}= & (4 p W z-(4 p-2) N) y, \\
\dot{z}= & (d-2 p-1) W+((4 p+2-d) N-\kappa) z-2 p W z^{2},
\end{aligned}
$$

and the constraints

$$
2 \kappa N+3-d+(d-5) N^{2}-\left(z^{2}-\frac{d-2 p-1}{2 p}\right) y=0
$$




$$
\begin{aligned}
& W^{2}-1-r T=0 \\
& T^{2 p}-r^{2 p-2} y=0 .
\end{aligned}
$$

\section{$4 \quad$ Fixed Points of the $F(2 p)$ model}

\section{1 $r=0$ and $r=\infty$}

Looking for globally regular solutions existing for $0 \leq r<\infty$ we have to cope with the singularities of Eqs. (13) at $r=0$ and $r=\infty$. Since for regular solutions the space time is locally flat at $r=0$ and $r=\infty$, we have to require $N=\kappa=1$ there. This implies that the radial variable $\tau$ behaves locally like $\ln r$ and thus $\tau \rightarrow \mp \infty$ for $r \rightarrow 0$ resp. $r \rightarrow \infty$. With a suitable choice of new dependent variables both singular points become hyperbolic fixed points (f.p.s). From this viewpoint regular solutions correspond to the 'stable manifold' of the respective fixed point [15]. As in the case of the BM solutions regularity requires $W= \pm 1$ and $U=0$ at $r=0$ and $\infty$. Let us first turn to the point $r=0$. Although a Taylor series expansion using the 'Schwarzschild' coordinate $r$ as an independent variable yields essentially one arbitrary parameter $b$ from $W=1-b r^{2}+O\left(r^{4}\right)$ as in the case of the $F(2)$ theory 1, 14, the linearisation at the f.p. is slightly more difficult due to the appearance of the singular expression $V=\left(W^{2}-1\right)^{2} / r^{4}$ and the auxiliary variable $z$ in the equations.

For $W=N=\kappa=1$ the equation for $z$ has the f.p.s $z=1$ and $(2 p+1-d) / 2 p$. It turns out that $z=1$ for solutions regular at $r=0$, while $z=(2 p+1-d) / 2 p$ yields a singular mode. This suggests the introduction of the variables

$$
\bar{W}=\frac{W-1}{r^{2}}, \quad \bar{z}=\frac{z-1}{r}, \quad \bar{N}=\frac{N-1}{r}, \quad \text { and } \quad \bar{\kappa}=\frac{\kappa-1}{r} .
$$

While $\bar{z}, \bar{N}$, and $\bar{\kappa}$ vanish at $r=0$ the variable $\bar{W}$ has the finite limit $-b$, where $b$ is the parameter of the Taylor expansion. From Eqs. (13) we obtain

$$
\begin{aligned}
\dot{\bar{W}} & =O(r), \\
\dot{\bar{z}} & =-d \bar{z}+(4 p+1-d) \bar{N}-\bar{\kappa}+O(r), \\
\dot{\bar{N}} & =-\bar{N}+\bar{\kappa}+O(r), \\
\dot{\bar{\kappa}} & =(d-4)(d-5) \bar{N}-2 \bar{\kappa}+O(r) .
\end{aligned}
$$

Replacing the $\tau$ derivatives by $r d / d r=(1+O(r)) d / d \tau$ these equations take the form required by Prop. 1 of [14. It is straightforward to see that besides the regular $r^{2}$ mode for $W$ the linearisation yields a singular one behaving as $r^{(2 p+1-d) / p}$. The $(N, \kappa)$ system yields an eigenvalue $3-d$, and a second one $d-6$ incompatible with the constraint Eq. (14) $)$. Thus for $d>3$ the behaviour of $N$ and $\kappa$ at $r=0$ is determined through the non-linear terms in Eqs. (19). One 
finds

$$
\begin{aligned}
& N=1-\frac{\left(4 b^{2}\right)^{p}}{4 p} r^{2}+O\left(r^{4}\right), \\
& \kappa=1+(4 p-3) \frac{\left(4 b^{2}\right)^{p}}{4 p} r^{2}+O\left(r^{4}\right) .
\end{aligned}
$$

The linearisation for $r \rightarrow \infty$ proceeds along similar lines. One finds that regular solutions behave like

$$
W= \pm\left(1-c r^{(2 p+1-d) / p}+\ldots\right)
$$

and there is a singular $r^{2}$ mode. Thus the regular and singular modes of $W$ exchange their role going from $r=0$ to $r=\infty$ as to be expected from $\tau \rightarrow \pm \infty$. Similarly the eigenvalue $3-d$ from the $(N, \kappa)$ system now yields a regular mode

$$
N=1-\frac{m}{2 r^{d-3}}+\ldots
$$

and solutions regular at $r=\infty$ are described by two free parameters, $c$ and the 'mass' $m$.

\section{2 $W=U=0$}

Besides the f.p.s for $r=0$ and $r=\infty$ there is also a f.p. for finite $r=r_{0}$ with $W=U=N=0$ and $\kappa=\kappa_{0}$. This f.p. is characteristic for the extremal Reissner-Nordstrøm solution at its horizon and therefore we call it the RN f.p. ${ }^{2}$

From the constraints Eqs. (14) we find

$$
r_{0}^{2-4 p}=\frac{2 p}{(d-2 p-1)(d-3)}, \quad \text { and } \quad \kappa_{0}^{2}=(2 p-1)(d-3) \text {. }
$$

Putting $r=r_{0}(1+\delta r), r U=\bar{U}$ and $\kappa=\kappa_{0}+\delta \kappa$ and keeping only linear terms we obtain from Eqs. (11) the system (eliminating $\delta r$ via Eq. (14))

$$
\begin{aligned}
& \dot{N}=\kappa_{0} N, \\
& \dot{\delta} \kappa=-2 \kappa_{0} \delta \kappa-(d+4 p-8) \kappa_{0} N, \\
& \dot{W}=\bar{U} \\
& \dot{\bar{U}}=-(d-2 p-1) W-\kappa_{0} \bar{U} .
\end{aligned}
$$

\footnotetext{
${ }^{2}$ The RN field configuration arising in the $d=4$ EYM theory is the infinite node limit of the regular solutions, in the external region the function $W(r)$ vanishing everywhere. In that case the $S O_{ \pm}(4)=S U_{ \pm}(2)$ gauge group breaks down to $S O(2)=U(1)$, namely this field configuration is an Abelian embedding. In the present cases, the $W(r)=0$ configuration of a $S O_{ \pm}(N)$ gauge field with $N \geq 5$, describes a $S O(N-1)$ gauge field, namely a non-Abelian embedding.
} 
The eigenvalues of these linear equations are $\kappa_{0},-2 \kappa_{0}$ for the $(N, \kappa)$ system and

$$
\lambda_{ \pm}=\frac{1}{2}\left(-\kappa_{0} \pm \sqrt{(2 p-5) d+2 p+7}\right),
$$

for the $(W, \bar{U})$ system. The eigenvalues $\lambda_{ \pm}$are always real for $p>2$, while for $p=1$ and $p=2$ they are real only if $d \leq 3$ resp. $d \leq 11$.

There is a similar f.p. with $N=\kappa=1$ and $W=U=0$ already present for the flat $F(2 p)$ model. Since then $\tau \approx \ln r$ this f.p. requires $r \rightarrow 0$ or $r \rightarrow \infty$. The corresponding eigenvalues $\lambda_{ \pm}$are

$$
\lambda_{ \pm}=\frac{1}{2}\left(4 p+1-d \pm \sqrt{(d-4 p-3)^{2}-8 p-4}\right) .
$$

These eigenvalues are real if $d$ lies outside the interval $[4 p+3-2 \sqrt{2 p+1}, 4 p+$ $3+2 \sqrt{2 p+1}]$. For $p=2$ this interval is $[6.528,15.472]$ and for $p=3$ it is $[9.709,20.292]$. As long as the eigenvalues have non-vanishing imaginary part the solutions coming close to the f.p. are oscillating. As a consequence we might expect to find regular solutions with any number of zeros. However, for $d>4 p+1$ the real part of $\lambda_{ \pm}$becomes negative and thus the solutions coming close to the f.p. tend to zero for $r \rightarrow \infty$ and $W$ cannot reach \pm 1 . In order to analyse these oscillating solutions beyond the linear approximation we consider the 'Lyapunov Function'

$$
Z=\left(z^{2}-\frac{d-2 p-1}{2 p}\right) r^{4 p-2} y=\left(W^{2}-1\right)^{2 p-2} \dot{W}^{2}-\frac{d-2 p-1}{2 p}\left(W^{2}-1\right)^{2 p},
$$

which is a measure of the amplitude of oscillations (as long as $|W|<1$ ), and satisfies

$$
\dot{Z}=2((4 p+2-d) N-\kappa)\left(W^{2}-1\right)^{2 p-2} \dot{W}^{2} .
$$

Thus for asymptotically flat solutions with $N \rightarrow 1, \kappa \rightarrow 1$ as $r \rightarrow \infty$, the amplitude of oscillations decreases for $d>4 p+1$, increases for $d<4 p+1$, and has a finite limit for $d=4 p+1$. That means that regular solutions with many zeros cease to exist for $d \geq 4 p+1$. However, since $4 p+3-2 \sqrt{2 p+1}<4 p+1$ there is always a finite $d$ interval, where regular solutions with any number of zeros are expected to exist.

\subsection{Conical singularity}

For $p>1$ there is another f.p., which turns out to be relevant for the limit $b \rightarrow \infty$ in certain dimensions (e.g. $d=5$ for the $F(2)+F(4)$ model). It is characterised by $W=1$ and nontrivial $(\neq 1)$ finite values of $N, \kappa$ and $z$. Putting the r.h.s. of Eqs. (16) to zero yields the f.p. equations

$$
0=\kappa N-N^{2}-y z^{2},
$$




$$
\begin{aligned}
0= & 1-\kappa^{2}+\frac{(d-4)(d-5)}{2}\left(N^{2}-1\right)+\frac{(4 p+2-d)}{2} y z^{2} \\
& +\frac{(d-2 p-1)(d+4 p-8)}{4 p} y, \\
0= & (4 p z-(4 p-2) N) y, \\
0= & (4 p+2-d) N z-\kappa z+d-2 p-1-2 p z^{2},
\end{aligned}
$$

leads to the solution

$$
\begin{aligned}
N_{0}^{2} & =\frac{16 p^{3}-8 d p^{2}+2 p d^{2}-8 p d+14 p+d-3 \pm \sqrt{Q}}{2\left(4 p^{2}-4 p+1\right)(2 p-1)} \\
\kappa_{0} & =(2 p+3-d) N_{0}+\frac{2 p(d-2 p-1)}{(2 p-1) N_{0}} \\
y_{0} & =\frac{4 p^{2}}{(2 p-1)^{2}}\left(\frac{\kappa_{0}}{N_{0}}-1\right) \\
z_{0} & =\frac{2 p-1}{2 p} N_{0}
\end{aligned}
$$

where

$$
Q=(2 p d-6 p+1)(d-3)\left(2 p d^{2}-16 p^{2} d-4 p d+d+32 p^{3}+10 p-3\right) .
$$

The zeros of the polynomial $Q$ are at $d=(6 p-1) / 2 p, 3$, and $\left(16 p^{2}+4 p-\right.$ $\left.1 \pm \sqrt{128 p^{3}-96 p^{2}+16 p+1}\right) / 4 p$. In order to obtain a real value for $N_{0}$ in the admissible region the dimension $d$ has to be restricted to the interval $3 \leq d \leq d_{p}$ with

$$
d_{p}=\frac{16 p^{2}+4 p-1-\sqrt{128 p^{3}-96 p^{2}+16 p+1}}{4 p},
$$

or it has to be larger than $\left(16 p^{2}+4 p-1+\sqrt{128 p^{3}-96 p^{2}+16 p+1}\right) / 4 p$. The numerical values of $d_{p}$ for $p=2,3$, and 4 are $\approx 5.63,8.63$, and 11.81 .

The fact that $N$ has a finite non-vanishing limit $N_{0}<1$ implies that $r \rightarrow 0$ for $\tau \rightarrow-\infty$. As a consequence of $N_{0} \neq 1$ the gravitational field has a 'conical' singularity at $r=0$ with a solid angle deficit and the YM field has a weak singularity

$$
W=1-\frac{1}{2} y_{0}^{1 / 2 p} r^{2-1 / p}+O\left(r^{2+1 / p}\right),
$$

as for the massive EYM model ('Proca theory') studied in 13 .

In order to linearise the Eqs. (16) at this 'conical' f.p. we put $N=N_{0}+\delta N$, $\kappa=\kappa_{0}+\delta \kappa, y=y_{0}+\delta y, z=z_{0}+\delta z$ and keep only linear terms in $\delta N$ etc. Eliminating $\delta \kappa$ via the constraint Eq. (17) we obtain

$$
\dot{\delta}=(3-d) N_{0} \delta N-\left(\frac{z_{0}^{2}}{2}+\frac{d-2 p-1}{4 p}\right) \delta y-y_{0} z_{0} \delta z
$$




$$
\begin{aligned}
\dot{\delta y}= & -2(2 p-1) y_{0} \delta N+4 p y_{0} \delta z \\
\dot{\delta z}= & \frac{2 p-1}{2 p}\left((4 p-3) N_{0}+\kappa_{0}\right) \delta N+\frac{2 p-1}{4 p}\left(\frac{d-2 p-1}{2 p}-z_{0}^{2}\right) \delta y \\
& +\left((4-d) N_{0}-\kappa_{0}-\frac{2 p-1}{2 p} y_{0} z_{0}\right) \delta z .
\end{aligned}
$$

Due to the complicated structure of this linear system its eigenvalues can only be determined numerically. For small $p$ one finds that there is one pair of complex conjugate eigenvalues if $d$ is near $d=2 p$, but there are three real ones if $d$ is larger than some value $d_{p}^{(r)}$. For $p=2$ resp. $p=3$ one finds $d_{2}^{(r)} \approx 5.55$ and $d_{3}^{(r)} \approx 8.0$. For $p \approx 5.2$ the value $d_{p}^{(r)}$ becomes equal to $2 p$ and all three eigenvalues are real for all $d \geq 2 p$. For $d=d_{p}$ one of the three negative modes becomes a zero mode, because the two different solutions for $N_{0}^{2}$ degenerate. As long as there are complex eigenvalues the solutions coming close to the f.p. oscillate.

Since the real parts of all three eigenvalues are negative for $d<d_{p}$ the f.p. is always repulsive for $\tau \rightarrow-\infty$. Thus there are no free parameters available that would be necessary to construct global solutions regular at $r=\infty$ with the behaviour (33) near $r=0$.

\section{$5 \quad F(2 p)+F(2 q)$ model; $q>p$}

Next we study the model with with the two terms $F(2 p)+F(2 q)$. As mentioned above we require $d>2 q$ (and hence $d>2 p+2$ ) and $d<4 q+1$. After a suitable rescaling this model depends on a single dimensionless parameter $\alpha$.

From Eqs. (11) we get

$$
\begin{aligned}
\dot{r}= & r N \\
\dot{W}= & r U, \\
\dot{N}= & (\kappa-N) N-\alpha^{2} V^{p-1}\left(1+V_{p q}\right) U^{2} \\
\dot{\kappa}= & 1-\kappa^{2}+\frac{(d-4)(d-5)}{2}\left(N^{2}-1\right) \\
& +\frac{\alpha^{2}}{2} V^{p-1}\left[\left(4 p+2-d+(4 q+2-d) V_{p q}\right) U^{2}+\left(\frac{(4 p-8+d)(d-2 p-1)}{2 p}\right.\right. \\
& \left.\left.+\frac{(4 q-8+d)(d-2 q-1)}{2 q} V_{p q}\right) T^{2}\right], \\
\dot{U}= & \frac{1}{1+V_{p q}}\left[((4 p+1-d) N-\kappa) U+((4 q+1-d) N-\kappa) V_{p q} U\right. \\
& \left.+W T\left(d-2 p-1+(d-2 q-1) V_{p q}-2\left(p-1+(q-1) V_{p q}\right) \frac{U^{2}}{T^{2}}\right)\right]
\end{aligned}
$$


and the constraint

$$
\begin{aligned}
2 \kappa N+3-d+(d-5) N^{2}= & \alpha^{2} V^{p-1}\left[\left(1+V_{p q}\right) U^{2}-\left(\frac{d-2 p-1}{2 p}\right.\right. \\
& \left.\left.+\frac{(d-2 q-1)}{2 q} V_{p q}\right) T^{2}\right]
\end{aligned}
$$

with $V_{p q}=V^{q-p}$.

Again it turns out to be useful to introduce the variables $y=V^{p-1} T^{2}$ and $z=U / T$ and rewrite the equations as

$$
\begin{aligned}
\dot{r}= & r N \\
\dot{W}= & r T z \\
\dot{N}= & (\kappa-N) N-\alpha^{2}\left(1+V_{p q}\right) y z^{2} \\
\dot{\kappa}= & 1-\kappa^{2}+\frac{(d-4)(d-5)}{2}\left(N^{2}-1\right)+\frac{\alpha^{2}}{2}\left[\left(4 p+2-d+(4 q+2-d) V_{p q}\right) z^{2}\right. \\
& \left.+\left(\frac{(4 p-8+d)(d-2 p-1)}{2 p}+\frac{(4 q-8+d)(d-2 q-1)}{2 q} V_{p q}\right)\right] y \\
\dot{y}= & 4 p\left(W z-\frac{2 p-1}{2 p} N\right) y, \\
\dot{z}= & \frac{1}{1+}\left[((4 p+2-d) N-\kappa) z+((4 q+2-d) N-\kappa) V_{p q} z\right. \\
& \left.+W\left(d-2 p-1+(d-2 q-1) V_{p q}-2\left(p+q V_{p q}\right) z^{2}\right)\right]
\end{aligned}
$$

with the constraints

$$
\begin{aligned}
2 \kappa N+3-d+(d-5) N^{2}= & \alpha^{2}\left[\left(1+V_{p q}\right) z^{2}\right. \\
& \left.-\left(\frac{d-2 p-1}{2 p}+\frac{(d-2 q-1)}{2 q} V_{p q}\right)\right] y, \\
W^{2}-1-r T= & 0, \\
T^{2 p}-r^{2 p-2} y= & 0 .
\end{aligned}
$$

The behaviour of solutions regular at $r=0$ is now given by the expansions

$$
\begin{aligned}
& N=1-\alpha^{2}\left(\frac{\left(4 b^{2}\right)^{p}}{4 p}+\frac{\left(4 b^{2}\right)^{q}}{4 q}\right) r^{2}+O\left(r^{4}\right), \\
& \kappa=1+\alpha^{2}\left((4 p-3) \frac{\left(4 b^{2}\right)^{p}}{4 p}+(4 q-3) \frac{\left(4 b^{2}\right)^{q}}{4 q}\right) r^{2}+O\left(r^{4}\right) .
\end{aligned}
$$

The $F(2 p)$ and $F(2 q)$ terms in Eqs. (41) scale differently. In the limit $b \rightarrow \infty$ the $F(2 p)$ term decouples and one obtains the BM type solutions of the $F(2 q)$ 
model after the rescaling $b \rightarrow b \alpha^{\frac{2}{2 q-1}}$ and letting $\alpha \rightarrow 0$. Likewise for $b \rightarrow 0$ and $d<4 p+1$ one obtains the BM type solutions of the $F(2 p)$ model rescaling $b \rightarrow b \alpha^{\frac{2}{2 p-1}}$ and letting now $\alpha \rightarrow \infty$.

From the virial theorem we know that for $4 q+1>d>4 p+1$ there is a flat solution, obtainable in the limit $\alpha \rightarrow 0$; the corresponding parameter $b_{\text {flat }}$ diverges as $d \rightarrow 4 q+1$ and vanishes in the limit $d \rightarrow 4 p+1$.

For $d=4 p+1$ and $b \rightarrow 0$ the $F(2 q)$ and gravity terms both decouple and one obtains a rescaled flat $F(2 p)$ instanton. Linearising the equations for a perturbation of the instanton background yields a finite limit for $b^{4 p-2 q-1} \alpha^{2}$; thus $\alpha$ vanishes as $b \rightarrow 0$ if $4 p<2 q+1$ or diverges if $4 p>2 q+1$.

It is easy to see that for $r \rightarrow \infty$ the terms coming from $F(2 q)$ are subleading and hence the asymptotic behaviour is that of the $F(2 p)$ model.

Also the other f.p.s besides $r=0$ and $r=\infty$ discussed for the $F(2 p)$ model have counterparts here. Of particular relevance for the application to limiting solutions is the RN f.p. with $W=U=N=0$ and finite values of $r$ and $\kappa$ determined by the equations

$$
\begin{aligned}
0= & 3-d+\frac{\alpha^{2}}{2 r_{0}^{4 p-2}}\left(\frac{d-2 p-1}{p}+\frac{d-2 q-1}{2 q r_{0}^{4 q-4 p}}\right), \\
\kappa_{0}^{2}= & 1-\frac{(d-4)(d-5)}{2}+\frac{\alpha^{2}}{4 r_{0}^{4 p-2}}\left(\frac{(4 p-8+d)(d-2 p-1)}{p}\right. \\
& \left.+\frac{(4 q-8+d)(d-2 q-1}{2 q r_{0}^{4 q-4 p}}\right) .
\end{aligned}
$$

Except for $d=2 p+1$ or $d=2 q+1$ the resulting equation for $r_{0}$ can only be solved numerically.

Similarly there is the f.p. with $W=U=0, N=\kappa=1$ and $r \rightarrow \infty$ already present in the flat $p$ - $q$-model. However, for $r \rightarrow \infty$ only the $F(2 p)$ term survives so we can use the corresponding results of the previous section.

What remains is the conical f.p. of Eqs. (29). It turns out to become relevant for the limiting solutions as $b \rightarrow \infty$ in the interval $2 q<d<d_{q}$ (e.g. $d=5$ for the 1-2-model). The numerical analysis shows that for large values of $b$ the solutions regular at $r=0$ come very close to the f.p. (of the $F(2 q)$ model) within a very short $r$ interval of length $r_{0}=1 / b^{q}$. They consist of an 'interior' part between $r=0$ and $r \approx \sqrt{r_{0}}$ and an 'exterior' one extending from $r \approx \sqrt{r_{0}}$ to $r=\infty$ (see Figs. 11 and 2).

The interior part is dominated by the $F(2 q)$ terms in Eqs. (39) and the expansions Eqs. (41) suggest the introduction of the rescaled variable $\bar{r}=r / r_{0} \equiv r b^{q}$. In the limit $b \rightarrow \infty$ the globally regular solutions split into an interior part extending from the regular origin $\bar{r}=0$ to the conical f.p. at $\bar{r}=\infty$ and an exterior one extending from the f.p. at $r=0$ to $r=\infty$. The spacetime corresponding to the interior part of the solution has a conical singularity at $\bar{r}=\infty$ with a solid angle deficit much like for global monopoles [16]. 
Solutions for sufficiently large $b$ can be obtained by matching a rescaled interior solution to an exterior one in a domain where the linearisation at the f.p. is appropriate. Although the $F(2 p)$ terms are a small perturbation for the interior solution, this is true for the exterior one only near the f.p., while the $F(2 p)$ term becomes dominant for large $r$.

In order to describe the interior part we use the variable $\bar{r}=r b^{q}$ and introduce $\bar{T}=b^{q-1} T$, such that $y=T^{2 q} r^{2-2 q}=\bar{T}^{2 q} \bar{r}^{2-2 q}$. For large $b$ we get from $y=O(1)$ the estimate

$$
W-1 \approx \frac{r T}{2} \sim \bar{r}^{\frac{2 q-1}{q}} b^{1-2 q}
$$

Putting $Y=(N, y, z)$ we can linearise Eqs. (39)) at the f.p. with $Y=Y_{0}+\delta Y_{I}$ to obtain

$$
\bar{r} \frac{d}{d \bar{r}} \delta Y_{I}=L \delta Y_{I}+O\left(\delta Y_{I}^{2}\right)+O\left(\bar{r}^{\frac{2 q-1}{q}} b^{1-2 q}\right) .
$$

$L$ here is the matrix on the r.h.s. of Eqs. (34), with some rescaling due to the appearance of $\alpha^{2}$ in Eqs. (39). In the region $1 \ll \bar{r}^{1 / q} \ll b$ we can neglect the $O($.$) terms to get$

$$
\delta Y_{I}=K \bar{r}^{\lambda} \eta=K(b r)^{\lambda}
$$

with some constant matrix $K$ and constant vector $\eta$ ( $\lambda$ denoting the diagonal matrix of eigenvalues). In order that the exterior solution hits the f.p. for $\tau \rightarrow$ $-\infty$ we have to fine-tune three parameters. Since the solutions regular at $r=\infty$ depend only on the two parameters $c$ and $m$ introduced in Eqs. (21) and (22), we have to tune also $\alpha$. This determines the 'critical' value of $\alpha$ which in fact is a way to compute it more precisely than as a limit of regular solutions starting at $r=0$. We put $\xi=(\alpha, m, c)$ with $\xi_{0}$ the triple parametrising the solution running into the f.p. For $\xi=\xi_{0}+\delta \xi$ the solution misses the f.p. but comes close to it for $|\delta \xi| \ll 1$. Linearisation at the f.p., valid for $r \ll 1$, gives now for the exterior solution

$$
\delta Y_{E} \approx K r^{\lambda} M \delta \xi
$$

with some constant matrix $M$. Matching the interior with the exterior solution at some fixed $r \ll 1$ using these approximations gives

$$
M \delta \xi=b^{q \lambda} \eta .
$$

If we rescale the $\delta \xi$ 's with the smallest real part of the $\lambda$ 's, the rescaled quantities either tend to a limit for $b \rightarrow \infty$ if the eigenvalues are real, or they oscillate about the limiting value in case they are complex. This behaviour is well exhibited in Figs. 3 and 4 .

\section{$6 \quad$ Numerical results}

The numerical analysis of the $F(2)+F(4)$ model [8, 9] showed that for $d=5,6,7$, and 8 there are 1-parameter families of globally regular solutions labelled by the 
parameter $\alpha$. All these solutions resemble the lowest BM solution with one zero of the potential $W$. In contrast to the BM case no solutions with more than one zero were found. All the families have a maximal value $\alpha_{\max }$ and end at some 'critical' value $\alpha_{c}$, where the solutions cease to exist. While for $d=5,6,7$ a critical value $\alpha_{c}>0$ was obtained, it was suggested that $\alpha_{c}=0$ for $d=8$. Our aim is to explain what happens in the limiting cases, determine the values of $\alpha_{c}$ and to analyse the corresponding critical solutions. As already mentioned above it proves very helpful for this purpose to consider also non-integer values of $d$, interpolating between the physically relevant integer ones. This is made possible by the explicit $d$-dependence of the Eqs. (11). In fact we extended our study of the $F(2)+F(4)$ model to the whole interval $4<d<9$ for which globally regular solutions can be found. We find that for $4<d<5$ analogues of the discrete family of BM solutions with any number of zeros of $W$ exist, while for $5 \leq d<9$ only solutions with one zero were found.

Let us first discuss the results for $5 \leq d<9$. Varying the parameter $\alpha$ for a fixed $d$ one obtains a smooth 1-parameter family of solutions conveniently parametrised by the curves $b(d, \alpha)$, where $b$ is the parameter characterising the solutions at $r=0$. These curves start with finite $b$ at $\alpha=0$ and depending on $d$ either turn back to $\alpha=0$ after running through some maximum or end at some finite value $\alpha_{c}$ (see Fig. 5). Our aim is to characterise the limiting solutions on both endpoints of the curve.

The endpoint $b(d, 0)$ is obtained by switching off the gravitational field taking $\alpha \rightarrow 0$. A was discussed in Section 5 the corresponding solutions are those of the flat $F(2)+F(4)$ theory already found some time ago [17. Actually, this holds only for $d>4 p+1=5$, while for $d=5$ a slightly different limit has to be taken switching off also the $F(4)$ term. More precisely, we have to rescale $r \rightarrow r / \alpha$ and simultaneously $b \rightarrow \alpha^{2} b$. The resulting flat space solution is nothing but the 4 dimensional instanton given by $W=\left(1-\frac{1}{2} b r^{2}\right) /\left(1+\frac{1}{2} b r^{2}\right)$ considered as a static soliton of the $d=5$ theory. Exactly the same function yields 'instanton' solutions for the $F(2 p)$ model in $d=4 p+1$.

The other endpoints of the curves $b(d, \alpha)$, obtained for large values of $b$, are more difficult to explain. These are different for $d=5[9]$ and $d=6,7,8[8]$. As was already observed in [9] for $d=5$ there seems to be a critical value $\alpha_{c} \approx 0.6$ and there are obviously other turning points of $b(d, \alpha)$ besides the maximum $\alpha_{\max } \approx 0.75$. For large values of $b$ the solutions show a very distinctive behaviour. $N$ decreases very quickly to some value $N_{0} \approx 0.82, W$ stays close to one, while $T^{2} / r$ and $U^{2} / r$ tend to finite limits. We claim that the solution comes closer and closer to the conical f.p. (30) as $b \rightarrow \infty$. In Section 5 we gave an analytical description of these solutions leading to an asymptotic formula for the parameters $\alpha, c$, and $m$ for large $b$ and to an independent determination of $\alpha_{c}$. The value $\alpha_{c}=0.595965$ obtained this way agrees up to this precision with the value obtained with a regular solution for $b=1000$. Employing Eq. (30) for 
$d=5$ we get $N_{0}=\sqrt{2 / 3} \approx 0.8165$ well in accordance with our numerical result (see Fig. (1). Also the limiting value of $y^{1 / 4}=\left(1-W^{2}\right) / r^{3 / 2}$ read of from Fig. [2 agrees well with the analytical result $y_{0}^{1 / 4}=2 / \sqrt{3 \alpha_{c}} \approx 1.496$. Fig. [3 displays the behaviour of $b^{3}\left(\alpha_{c}-\alpha(b)\right)$ etc. for large values of $b$, which is in accordance with our derivation at the end of Section 5 . As also mentioned in Section 3, the f.p. ceases to be oscillatory at $d \approx 5.55$, which is in accordance with the behaviour shown in Fig. 团 for $d=5.56$.

The f.p. exists only for $d \leq d_{2}$ with $d_{2}=(71-\sqrt{673}) / 8 \approx 5.632$ given by Eq. (32). Thus this f.p can only be relevant for the limiting solution up to this value, again well in accordance with our numerical results as shown in Fig. 6]

For $d>d_{2}$ a different limiting behaviour manifests itself. As far as the numerical results for $d=6$ are concerned, the function $N$ seems to develop a double zero at some finite value of $r$, where the variable $\tau$ increases without limit. This type of critical behaviour is well known from the limiting solution of the BM family, when the number of zeros goes to infinity [14] and from gravitating monopoles as described in [18]. The critical solution runs into the RN f.p. and thus consists of a non-trivial (geodesically complete) interior solution and an exterior (geodesically incomplete) one with $W=0$ representing the exterior of the extremal RN black hole.

Instead of looking for globally regular solutions one may as well look for solutions starting at $r=0$ and ending at the RN f.p. Fig. 5 shows corresponding curves $b(d, \alpha)$ as dashed lines. As discussed in Section 5 we have to rescale $b \rightarrow b \alpha^{2 / 3}$ in order to obtain the BM solution of the $F(4)$ model. The same rescaling has to be chosen for the solution running into the RN f.p. For $d=6$ there seems to be a bifurcation of the globally regular solutions with these RN ones at $\alpha \approx 0.015$, although numerically it is clearly impossible to distinguish a genuine bifurcation from a finite but extremely small proximity. At any rate, it is rather obvious from Fig. [5 that the curve $b(d, \alpha)$ for $d=8$ extends all the way to $\alpha=0$ without any bifurcation with the $\mathrm{RN}$ curve, agreeing with the numerical results of $[8$. Although for $d=7$ this is not obvious from Fig. 5 our numerical results clearly exclude a bifurcation. In fact, numerically we can exclude a bifurcation down to $d \approx 6.8$. Thus one might expect some particular value $d=d_{b}$ for which the bifurcation occurs at $\alpha=0$ and then for $d<d_{b}$ moves along some curve $\alpha(d)$ down to $d=d_{2}$. In the following we shall give a convincing argument, that actually no such bifurcation happens for any $d_{2}<d<9$. This argument is based on an observation made for the BM solutions with many zeros, i.e. close to the limiting solution running into the RN f.p. 14. There, solutions with $n \gg 1$ zeros show three characteristic regions. Region I extends from $r=0$ to $r=1$, where the solutions develop more and more zeros of $W$ and come close to the oscillating limiting solution running into the RN f.p., for which $W$ decays like $e^{-\tau / 2}$ for $\tau \rightarrow \infty$. In region II, between $r=1$ and $r=r_{n} \gg 1$ the functions $W$ and $U$ first continue to decay, but then they start to grow like $e^{\tau / 2}$, however, 
still staying very small. In this interval the metric is well approximated by the extremal RN solution growing from $N \approx 0$ at $r=1$ to $N \approx 1$ for $r \gg 1$. Thus the solution is well approximated solving the linearised equation for $W$ and $U$ in the background of the extremal RN solution. Finally, in region III between $r=r_{n}$ and $r=\infty$ the solutions stay close to the oscillating flat space solution for $W$ running from $r=\infty$ into the f.p. with $U=W=0$ and $N=\kappa=1$. The relevant difference between the RN f.p. of the $(U, W)$ system with $N=0$ and the flat one with $N=1$ manifests itself in the real part of the eigenvalue being $-1 / 2$ for $N=0$ and $+1 / 2$ for $N=1$.

In the present case, if we assume a bifurcation with the solution running into the RN f.p. the same structure with three regions must form. Now we will argue that this leads to a contradiction. For $\alpha \neq 0$ the flat f.p. with $W=0$ is dominated by the $p=1$ term at large $r$ and thus is oscillatory in the interval $|d-7| \leq 2 \sqrt{3} \approx 3.46$. Since we are considering a family of solutions with one zero they cannot come close to this f.p. in the considered region with $d \approx 6$. This means that the curve $b(d, \alpha)$ has to turn back all the way to $\alpha=0$. There still remains the possibility that the bifurcation happens at $\alpha=0$ for some or all $d \leq d_{b}$. Since $b \rightarrow \infty$ as $\alpha \rightarrow 0$ it is the $F(4)$ theory that is relevant in this case. As found in Section 4 the eigenvalues at the flat f.p. with $W=0$ are real only if $d<11-2 \sqrt{5} \approx 6.528$, thus $d$ must obey this bound. Suppose there is some maximal value $d_{m}<6.528$ for which the $p=2$ solution runs into the RN f.p., then it must come arbitrarily close to it for slightly larger values of $d$. As described above, the function $W$ should then stay small in a large $r$-interval, while $N$ runs from $N \approx 0$ to $N \approx 1$. From Eqs. (2526) it follows that in the considered domain for $d$ there are always two convergent modes in the $(W, U)$ subsystem corresponding to two negative eigenvalues for the RN f.p. $(\tau \rightarrow+\infty)$ and two positive ones for the flat f.p. $(\tau \rightarrow-\infty)$. However for solutions coming very close to both f.p.s the modes with the eigenvalues of larger modulus are suppressed, i.e. near the RN f.p. it is $\lambda_{+}^{\text {RN }}$ that dominates, while it is $\lambda_{-}^{\text {flat }}$ at the flat f.p. The best way to visualise the corresponding change of the $(W, U)$ system moving from one f.p. to the other is to consider the quotient $\eta=r U / W$ obeying a Riccati equation in the linear approximation

$$
\dot{\eta}=5-d+((10-d) N-\kappa) \eta-\eta^{2}
$$

with $N$ and $\kappa$ given by the extremal $\mathrm{RN}$ solution

$$
N=\left(1-\frac{2 M}{r^{d-3}}+\frac{d-5}{4(9-d) r^{6}}\right)^{\frac{1}{2}}, \quad \text { with } \quad M=\frac{3}{(9-d)}\left(\frac{d-5}{4(d-3)}\right)^{\frac{d-3}{6}},
$$

and

$$
\kappa=\frac{d-3+(5-d) N^{2}-\frac{d-5}{4 r^{6}}}{2 N} .
$$

Supposing there is a solution with the correct behaviour at both f.p.s we should find a solution of the Riccati equation interpolating between $\lambda_{+}^{\mathrm{RN}}$ at the RN f.p. 
$\left(r=r_{0}\right)$ and $\lambda_{-}^{\text {flat }}$ at the flat one $(r \rightarrow \infty)$. For $d=6$ the Eq. (48) can be integrated in closed form resulting in a solution running from $\lambda_{+}^{\mathrm{RN}}$ to $\lambda_{+}^{\text {flat }}$ instead to $\lambda_{-}^{\text {flat}}$, excluding a bifurcation under the assumptions made. For other values of $d$ in the relevant interval $5.632<d<6.528$ numerical integration of Eq. (48) yields the same negative result. Therefore we conclude that the dashed and solid curves in Fig. 5 run back to different points at the $b$ axis, although numerically we are unable to find regular solutions below $d \approx 6.8$.

There remains the interval $4<d<5$ for which no regular flat solutions exist according to the virial theorem. On the other hand there are self-gravitating solutions of the $F(2)$ model generalising the BM solutions of $d=4$. In fact there are again families $b(d, \alpha)$ of regular solutions tending to these BM type solutions in the limit $b \rightarrow 0$ and $\alpha \rightarrow \infty$ after suitable rescalings as discussed in Section 5 . Fig. [6 shows some of the curves $b(d, \alpha)$. For $b \rightarrow \infty$ they tend to the conical f.p. as for $5 \leq d<d_{q}$.

In order to further support our results for the 1-2-model we have also performed some numerical calculations for the 2-3- and the 2-4-models. Figs. 7 and 8 show again agreement with our analytical results from Section 3 . For $d<4 p+1=9$ the curves $b(d, \alpha)$ have a BM type limit for $\alpha \rightarrow \infty$ after suitable rescaling, while for $d>9$ they start from $\alpha=0$ with the flat space solutions with finite values of $b$. For $d<d_{3} \approx 8.63$ resp. $d<d_{4} \approx 11.81$ the curves approach a finite limit for $\alpha$ as $b \rightarrow \infty$ and the solutions run into the conical f.p., while for larger values of $d$ the curves $b(d, \alpha)$ run back to $\alpha=0$ approaching the BM type solutions of the $F(6)$ resp. $F(8)$ model after rescaling.

The results on the f.p.s with $W=0$ and $N=0$ resp. $N=1$ have another interesting consequence. Solutions of the $p$ - $q$-model, which come close to the RN f.p. and for which $N \rightarrow 1$ for large $r$ come also close to the flat f.p. of the $F(2 p)$ model with $W=0$ (for $r \gg 1$ the $F(2 p)$ terms dominate those of the $F(2 q)$ part). Although the RN f.p. is non-oscillatory for $p>2$, the flat one is oscillatory in the interval $4 p+3-2 \sqrt{2 p+1}<d<4 p+3+2 \sqrt{2 p+1}$ with positive real part as long as $d<4 p+1$. Thus we expect to find regular solutions with any number of zeros of $W$ accumulating at $r \rightarrow \infty$ for $4 p+3-2 \sqrt{2 p+1}<d<4 p+1$. For $p=1$ this interval is $3.54<d<5$ (remember, however, that we assume $d \geq 4$ ), while for $p=2$ we get $6.528<d<9$. This is compatible with the observation of [8] that no multi-node solutions exist for the 1-2-model for $d \geq 5$. On the other hand for the 2-3-model multi-node solutions are expected to exist for the integer dimensions $d=7$ and $d=8$ and were actually found in our numerical analysis. Figs. 9 and [0] show solutions of the $F(4)$ theory for $d=8$ with two and three zeros of $W$. Solutions for $d=7$ get much closer to the RN f.p. and are therefore more difficult to obtain numerically. Fig. 11 shows a solution of the 2-3-model for $d=7$ and $\alpha=20$ with two zeros of $W$.

Since the eigenvalues of the f.p. have negative real part for $d>4 p+1$ there are solutions with $W \rightarrow 0$ for $r \rightarrow \infty$ (see Fig. 12). 


\section{Summary}

Solutions to a family of EYM models in higher dimensions have been studied. These models involve higher order YM curvature terms $F(2 p)$ characterised by integers $p, p=1$ giving the standard YM theory. For reasons of simplicity we have restricted the number of such terms to two ( $p$ - $q$-models) depending on just one dimensionless parameter $\alpha$. Studying the corresponding one-parameter families of (static, spherically symmetric) solutions allows us to capture the qualitative properties of EYM solutions in higher dimensions. We have restricted our study, for simplicity, to regular solutions only, knowing that corresponding black hole solutions exist.

The simplest example of the family of theories we have considered (the 1-2model) was recently studied in [8, 9] devised specifically to suit spacetime dimensions $4<d<9$. The more general $p$ - $q$-models considered here suit also higher spacetime dimensions. The central part of the work here was aimed at understanding qualitatively the results found from the numerical analysis of [8, 9. This was achieved by analytic analysis supplemented with numerical studies including in addition to the previously considered case $p=1, q=2$ also the cases $p=2$, $q=3$ and $p=2, q=4$. The analytic part of the work was that of a fixed point analysis of nonlinear ordinary differential equations, employed previously [14, 13] for the familiar $d=4 \mathrm{BM}$ solutions and magnetic monopoles.

Like in the case of the gravitating monopoles 13 of the EYMH system, one finds families of solutions parametrised by the dimensionless parameter $\alpha$, which can be understood as a quotient of mass scales of the YM and the gravity parts of the theory. Since the $\alpha$ dependence of the solutions shows several distinctive behaviours for different dimensions it turned out to be particularly useful to vary the dimension parameter $d$ continously, which is possible due to the explicit dependence of the radial field equations on $d$. This trick allowed us to relate the changes with $d$ to changes in the fixed point structure of the equations. We find several different possibilities for the $\alpha$ dependence of the solutions that can be characterised by their behaviour for small $b$ and for large $b$ (see Figs. 6, (7) and 8). There are three possibilities for small $b$ :

- For $2 q<d<4 p+1$ the parameter $\alpha$ decreases from infinity as $b$ increases from zero. In the limit $\alpha \rightarrow \infty$ the solutions approach a suitably rescaled BM type solution of the $F(2 p)$ model.

- For $d=4 p+1$, as $b$ starts from zero the parameter $\alpha$ either increases from zero if $4 p<2 q+1$, or decreases from infinity if $4 p>2 q+1$. In the limit $b \rightarrow 0$ the solutions approach the instanton of the flat $F(2 p)$ model.

- For $4 p+1<d<4 q+1$ the parameter $\alpha$ increases from zero as $b$ starts from a finite value. For $\alpha \rightarrow 0$ the solutions approach a solution of the flat $p$ - $q$-model. 
And there are two possibilities for large $b$ :

- For $2 q<d \leq d_{q}$ the parameter $\alpha$ approaches a finite limiting value $\alpha_{c} \neq 0$ as $b \rightarrow \infty$. In the limit the solutions tend to a new type of fixed point, which we named 'conical' in view of the corresponding space-time singularity.

- For $d_{q}<d<4 q+1$ the parameter $\alpha$ decreases to zero as $b \rightarrow \infty$. For $\alpha \rightarrow 0$ the solutions approach the BM type solution of the $F(2 q)$ model. Note, however, that this limit cannot be explored numerically for values of $d$ close to $d_{q}$ because the solutions come too close to the RN f.p.

Clearly not all six combinations of these possibilities can be realised simultaneously for one $p$-q-model, because some of the constraints on them are mutually exclusive.

The existence of multi-node solutions is related to the existence of a f.p. with complex eigenvalues corresponding to a focal point. In the present case there is such a f.p. with vanishing YM potential $W$. Our analytical analysis reveals that for the $p$ - $q$-models multi-node solutions are expected for dimensions in the interval $4 p+3-2 \sqrt{2 p+1}<d<4 p+1$ perfectly compatible with our numerical results.

\section{Acknowledgements}

Thanks to Eugen Radu for useful discussions. We are grateful to the Alexandervon-Humboldt Foundation and the Dublin Institute for Advanced Studies for their support. This work was carried out in the framework of projects SC/03/390 and IC/05/03 of Enterprise-Ireland. 


\section{References}

[1] R. Bartnik and J. McKinnon, Phys. Rev. Lett. 61 (1988) 141.

[2] M.S. Volkov and D.V. Gal'tsov, JETP Lett. 50 (1989) 346.

[3] P. Bizon, Phys. Rev. Lett. 64 (1990) 2844.

[4] H.P. Künzle and A.K.M. Masood ul Alam, J. Math. Phys. 31 (1990) 928.

[5] M.B. Green, J.H. Schwarz and E. Witten, Superstring Theory, Cambridge University Press, Cambridge, 1987.

[6] J. Harvey and A. Strominger, TASI lectures on quantum aspects of black holes, hep-th/9209055.

[7] J. Polchinski, TASI lectures on D-branes, hep-th/9611050.

[8] Y. Brihaye, A. Chakrabarti and D.H. Tchrakian, Class. Quantum Grav. 20 (2003) 2765 hep-th/0202141.

[9] Yves Brihaye, A. Chakrabarti, Betti Hartmann and D.H. Tchrakian, Phys. Lett. B 561 (2003) 161 hep-th/0212288.

[10] A.A. Tseytlin, Born-Infeld action, suersymmetry and string theory, in Yuri Golfand memorial volume, ed. M. Shifman, World Scientific, 2000.

[11] E. Bergshoeff, M. de Roo and A. Sevrin, Fortsch. Phys. 49 (2001) 433-440; Nucl. Phys. Proc. Suppl. 102 (2001) 50-55.

[12] M. Cederwall, B. Nilsson and D. Tsimpis, JHEP 0106 (2001) 034.

[13] P. Breitenlohner, P. Forgacs and D. Maison, Nucl. Phys. B 383 (1992) 357; ibid. 442 (1995) 126.

[14] P. Breitenlohner, P. Forgács and D. Maison, Commun. Math. Phys. 163 (1994) 141

[15] P. Hartman, Ordinary Differential Equations, Boston: Birkhäuser, 1982.

[16] M. Barriola and A. Vilenkin, Phys. Rev. Lett. 63 (1989) 341.

[17] J. Burzlaff and D. H. Tchrakian, J. Phys. A 26 (1993) L1053.

[18] P. Breitenlohner, P. Forgacs and D. Maison, Nucl. Phys. B 442 (1995) 126. 


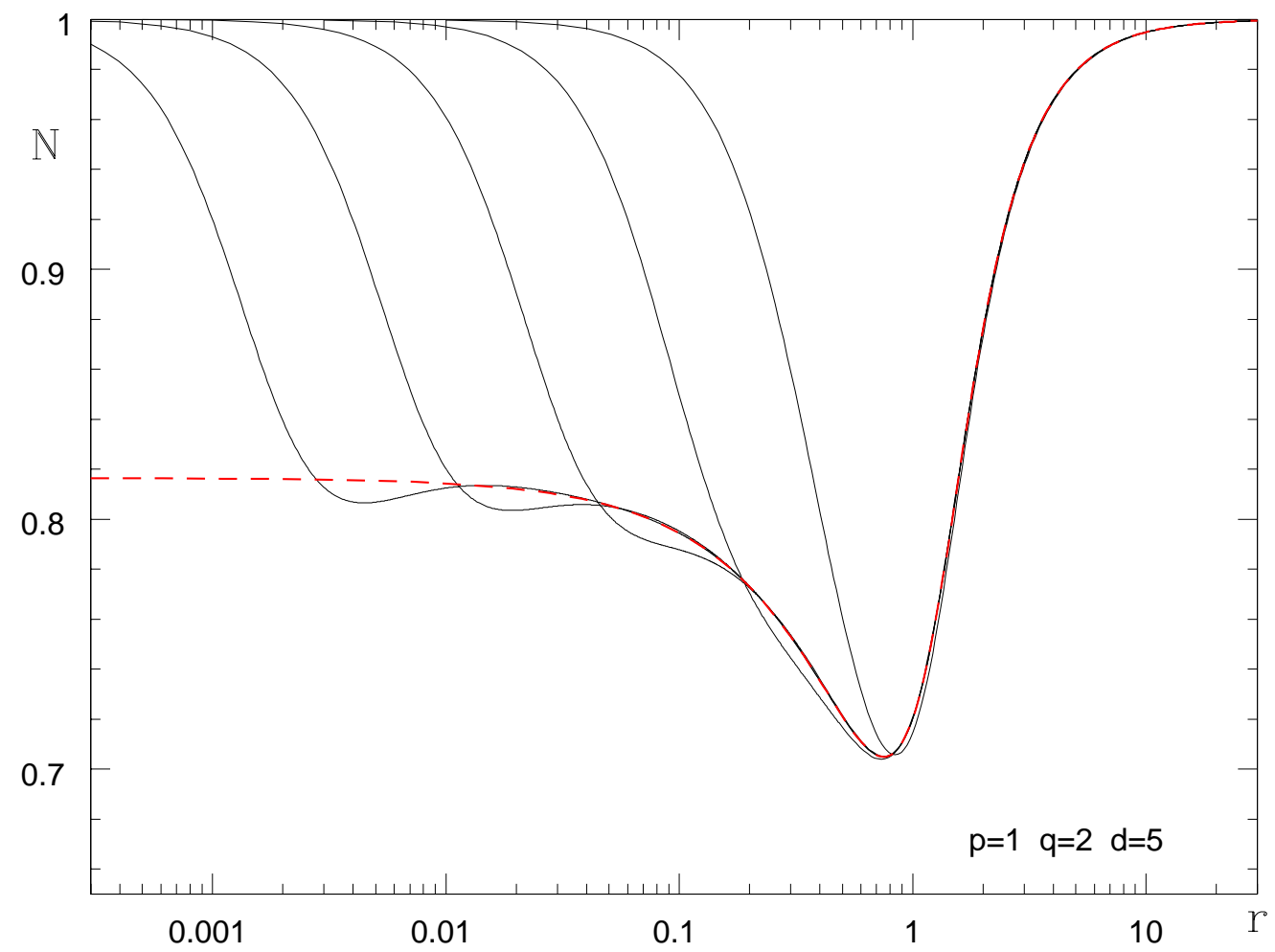

Figure 1: $N(r)$ of the 1-2-model for $d=5$ and increasing values of $b$ 


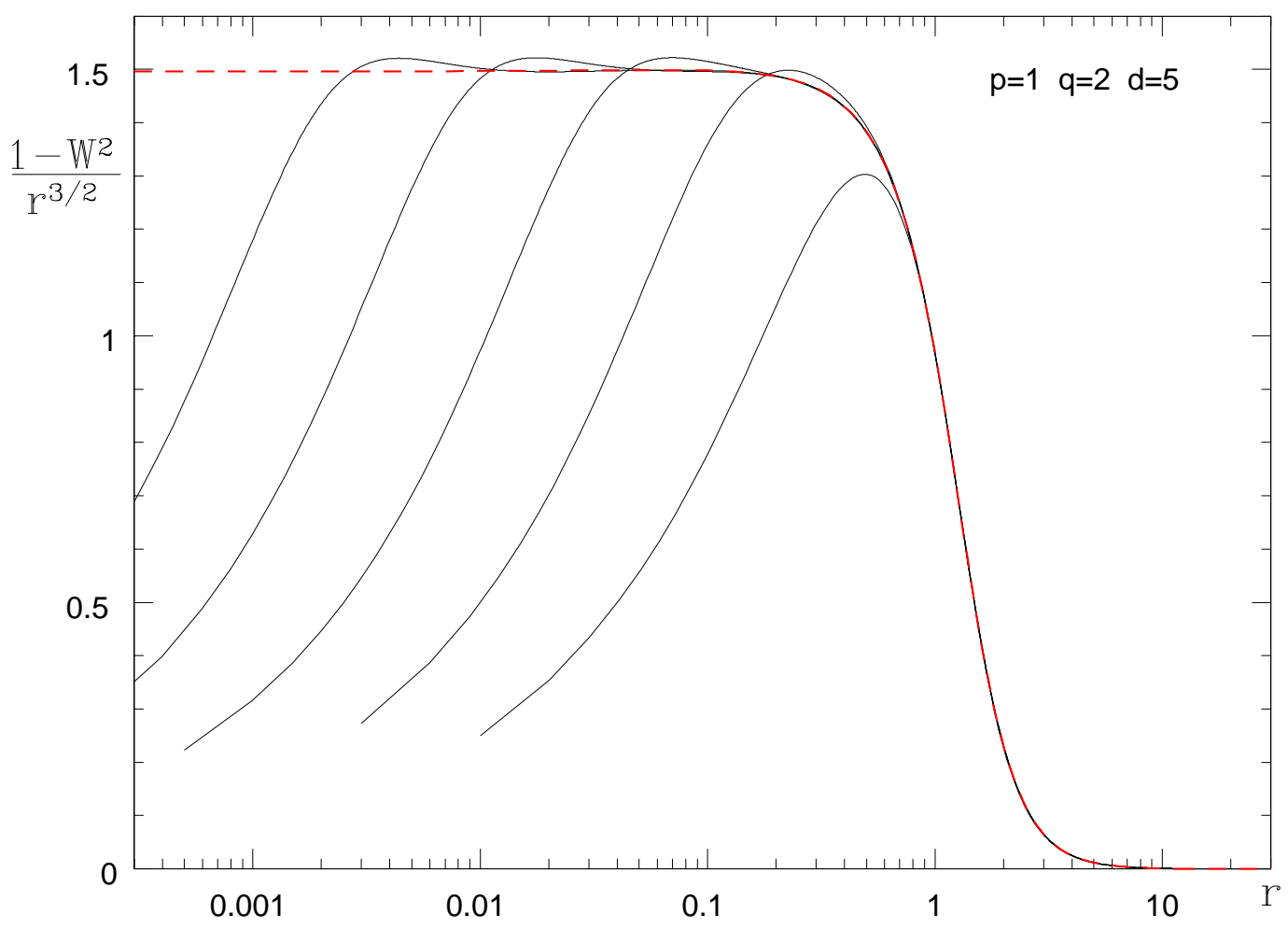

Figure 2: $y^{1 / 4}=\left(1-W^{2}\right) / r^{3 / 2}$ of the $1-2$-model for $d=5$ and increasing values of $b$ 


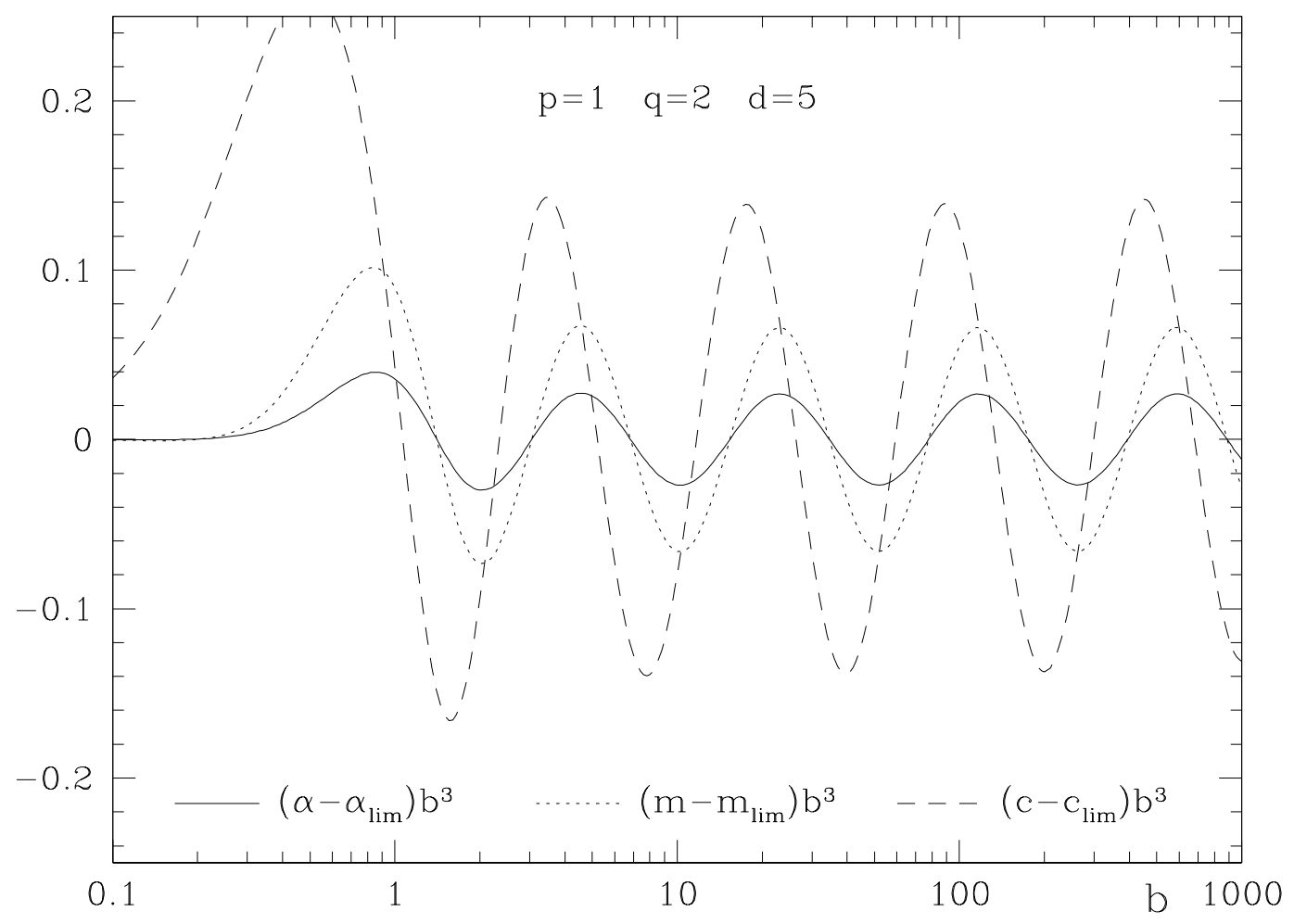

Figure 3: Rescaled parameters of the 1-2-model for $d=5$ and large $b$ 


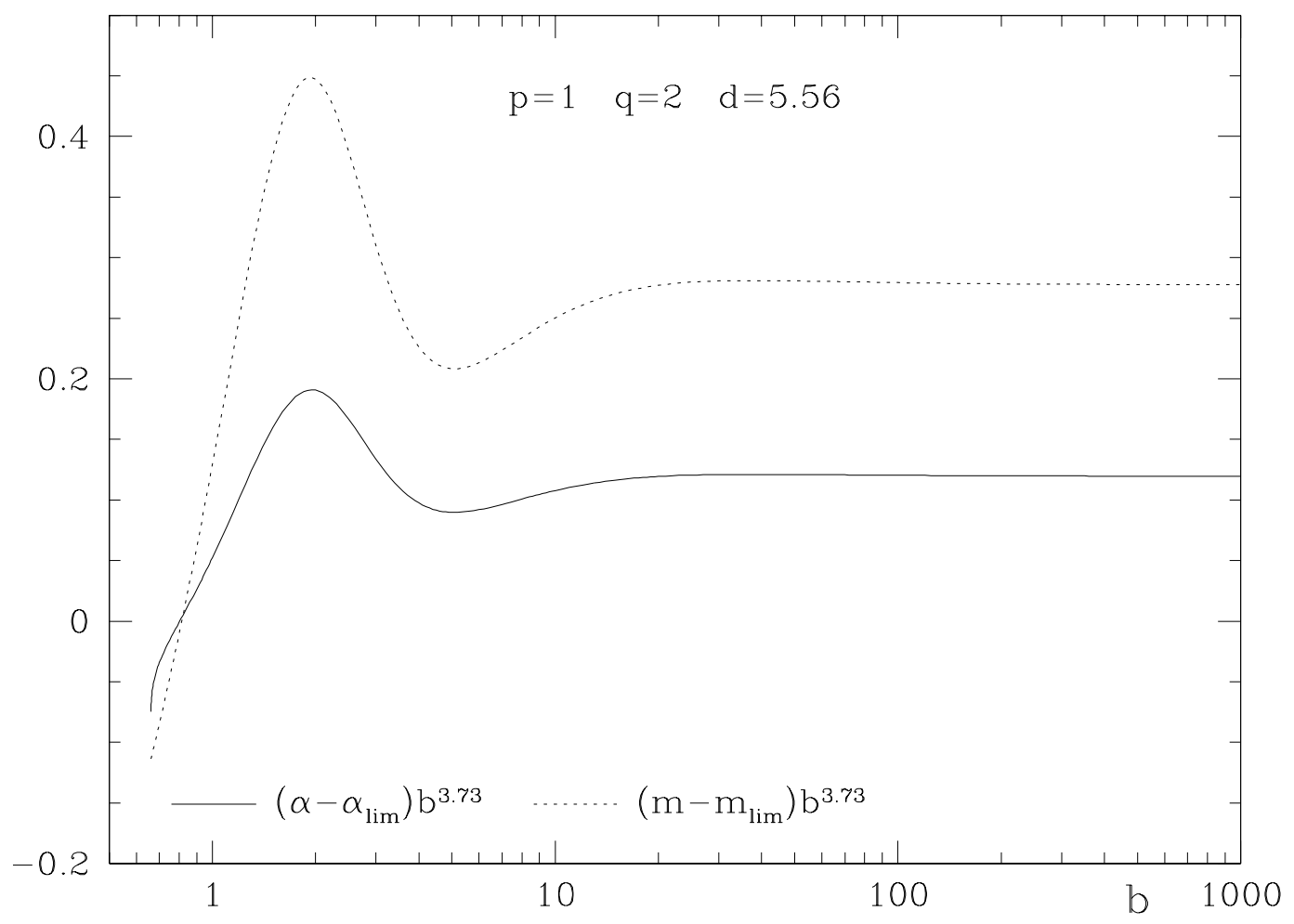

Figure 4: Rescaled parameters of the 1-2-model for $d=5.56$ and large $b$ 


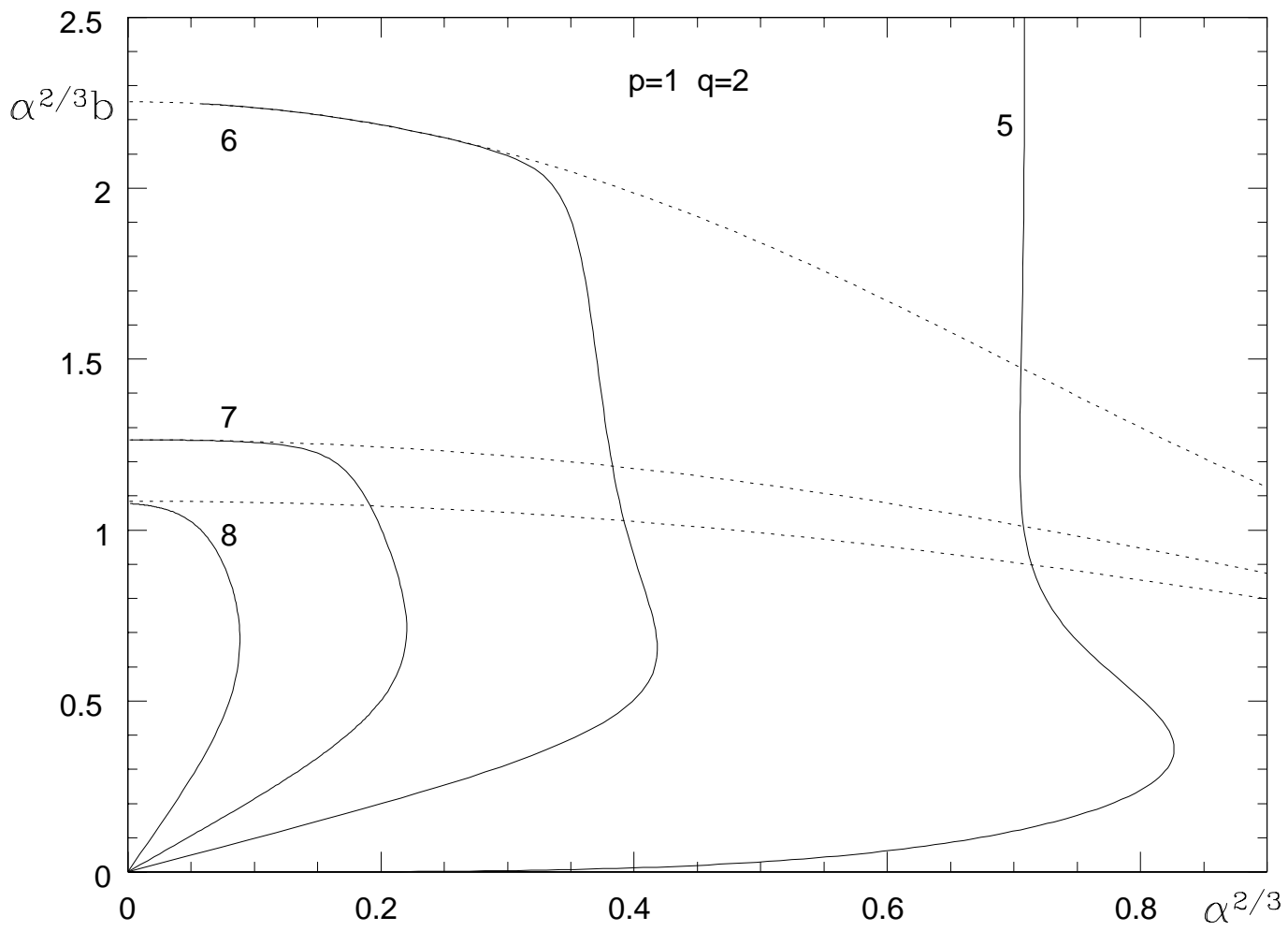

Figure 5: $b$ as a function of $\alpha$ for regular solutions of the 1-2-model with $d=5$, 6,7 , and 8 


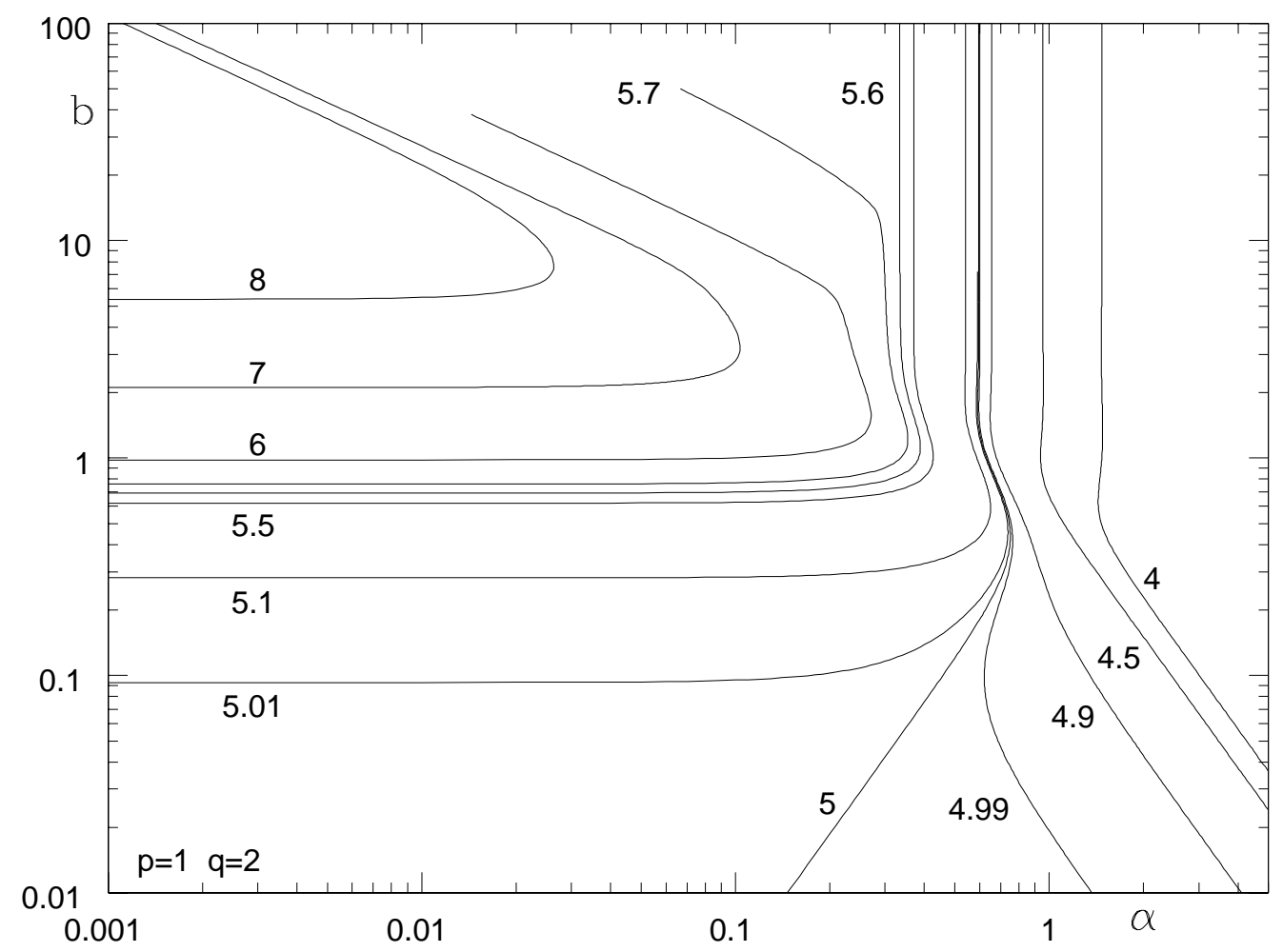

Figure $6: b$ vs. $\alpha$ for regular solutions of the 1-2-model with different values of $d$ 


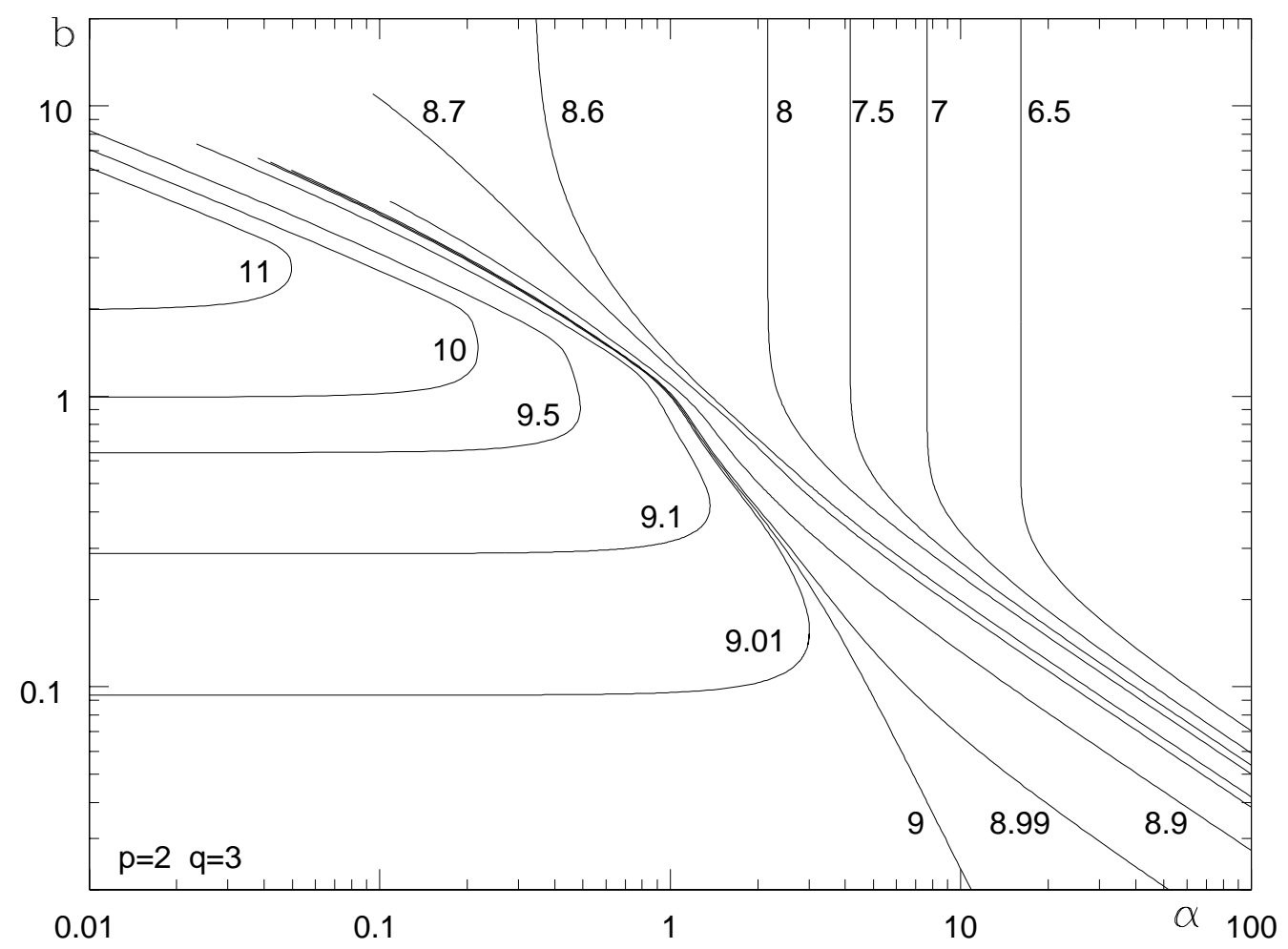

Figure 7: $b$ vs. $\alpha$ for regular solutions of the 2-3-model with different values of $d$ 


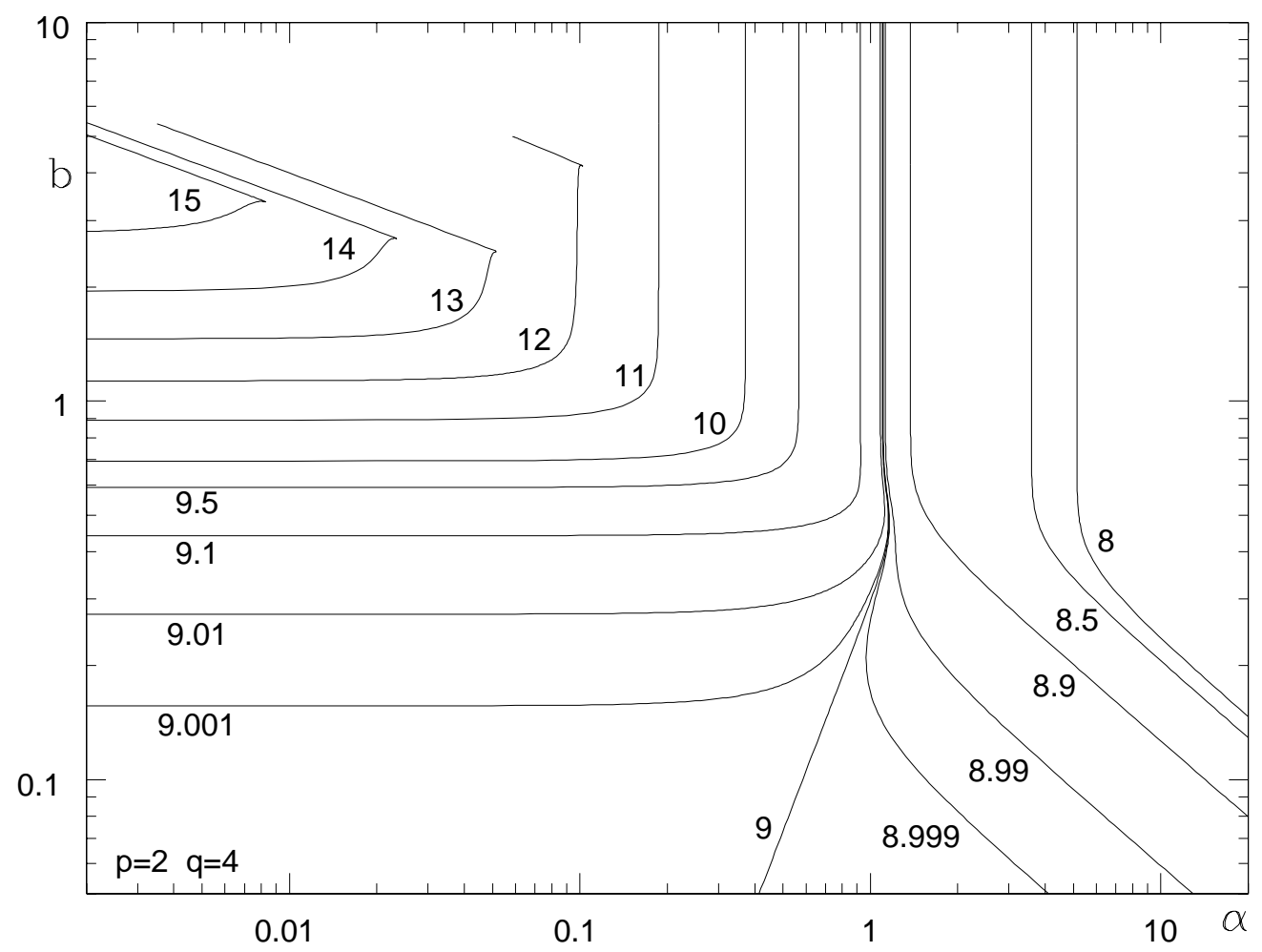

Figure 8: $b$ vs. $\alpha$ for regular solutions of the 2-4-model with different values of $d$ 


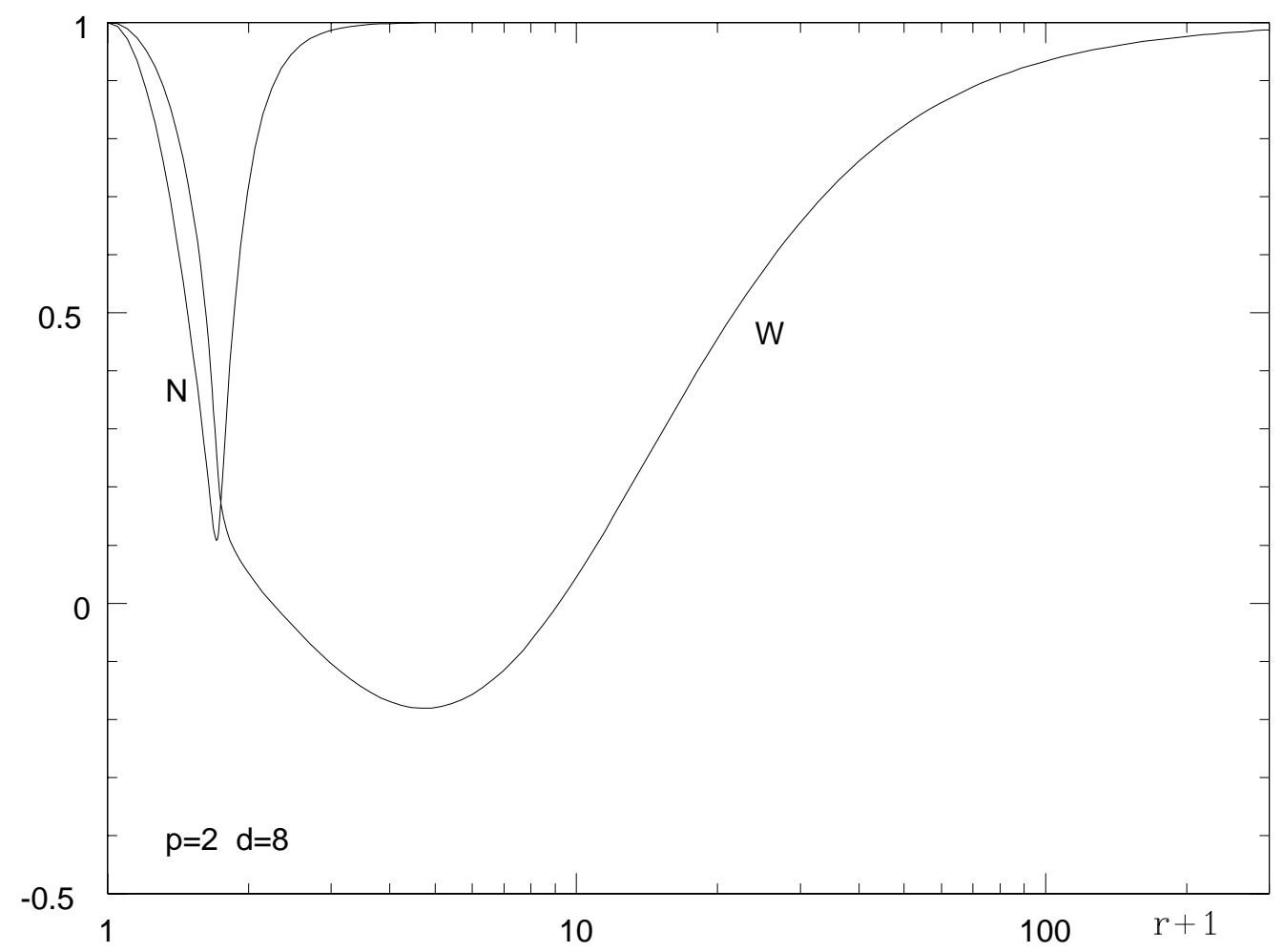

Figure 9: $p=2, d=8$ solution with two zeros of $W$ 


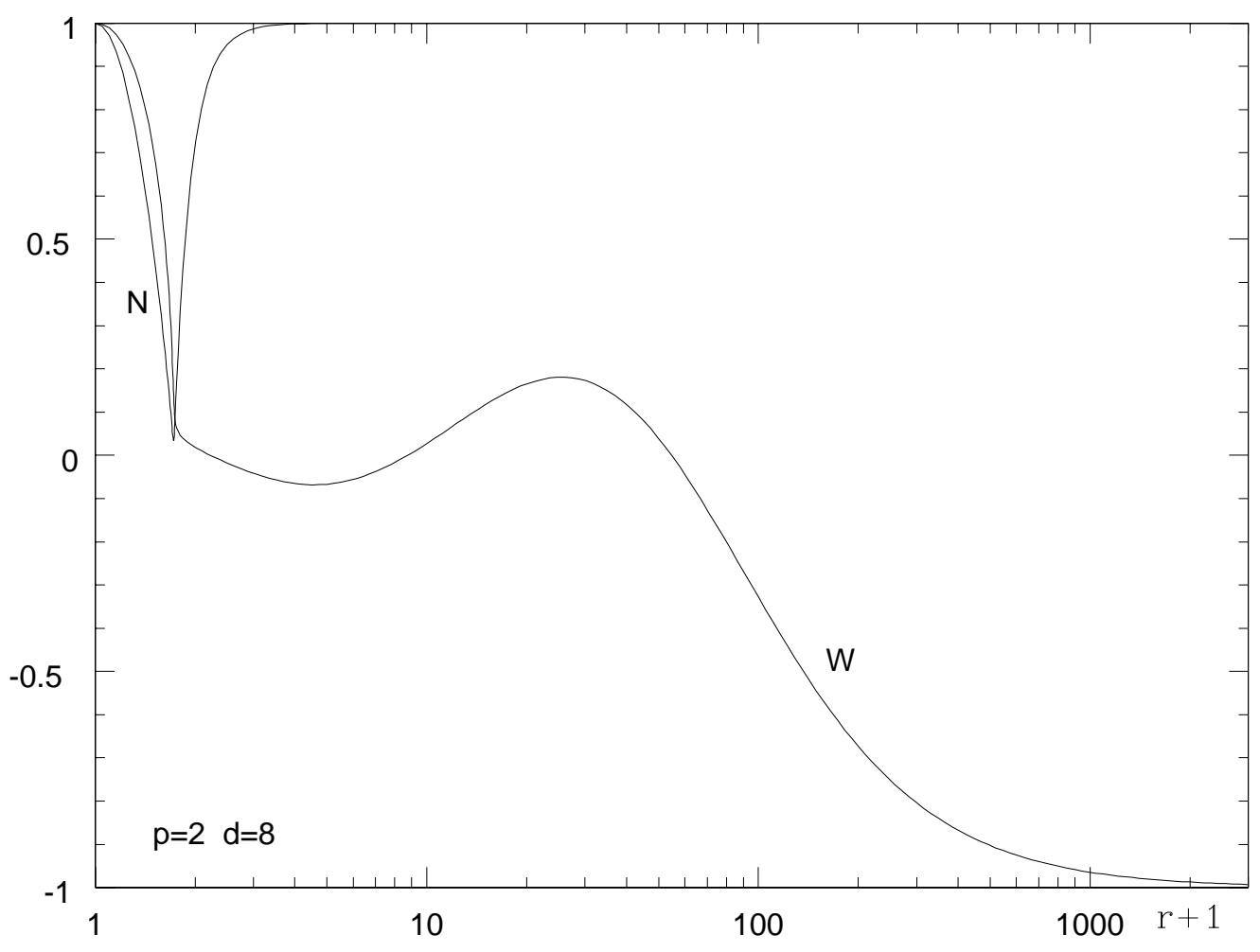

Figure 10: $p=2, d=8$ solution with three zeros of $W$ 


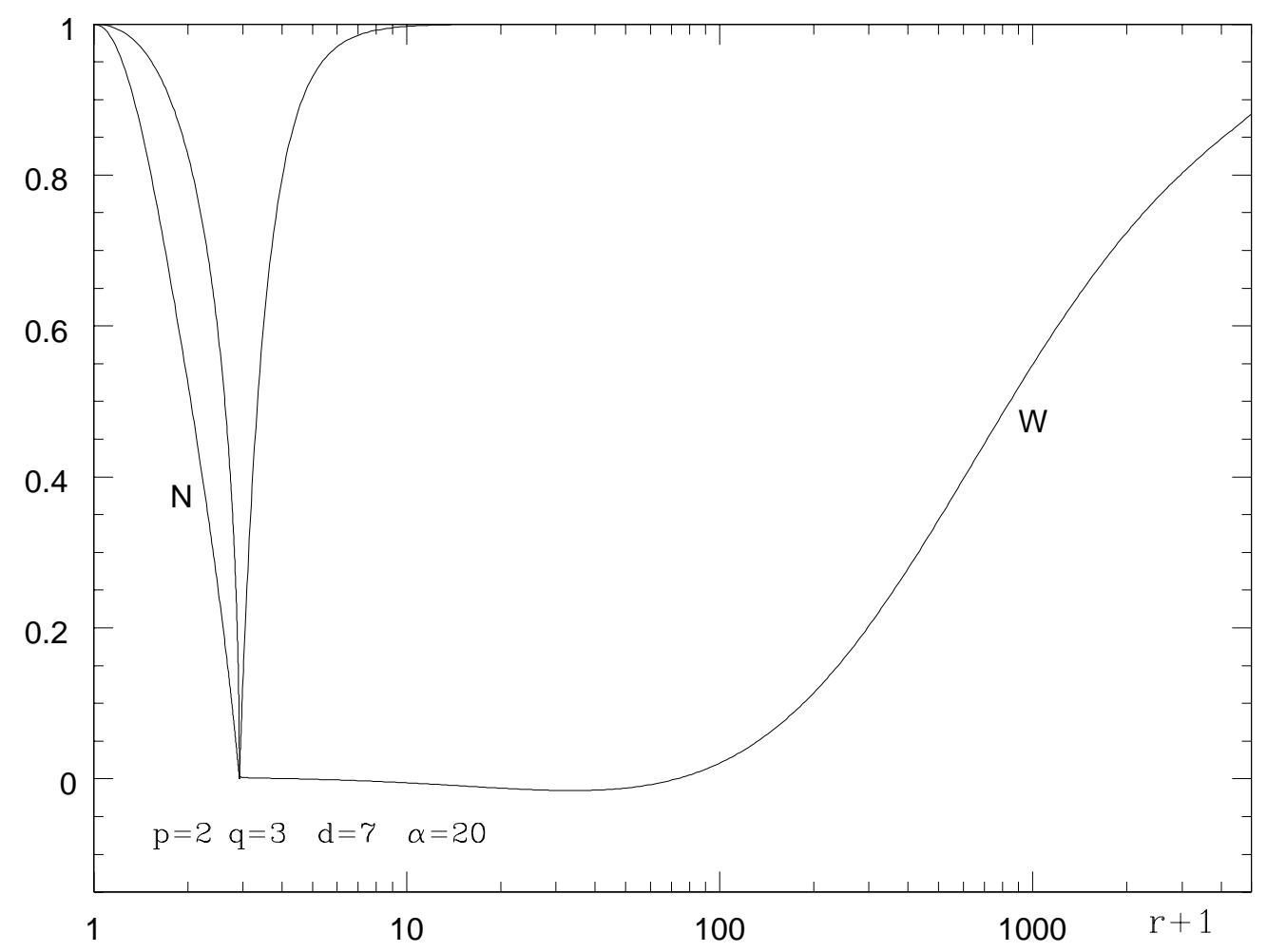

Figure 11: Solution of the 2-3-model for $d=7$ and $\alpha=20$ with two zeros of $W$ 


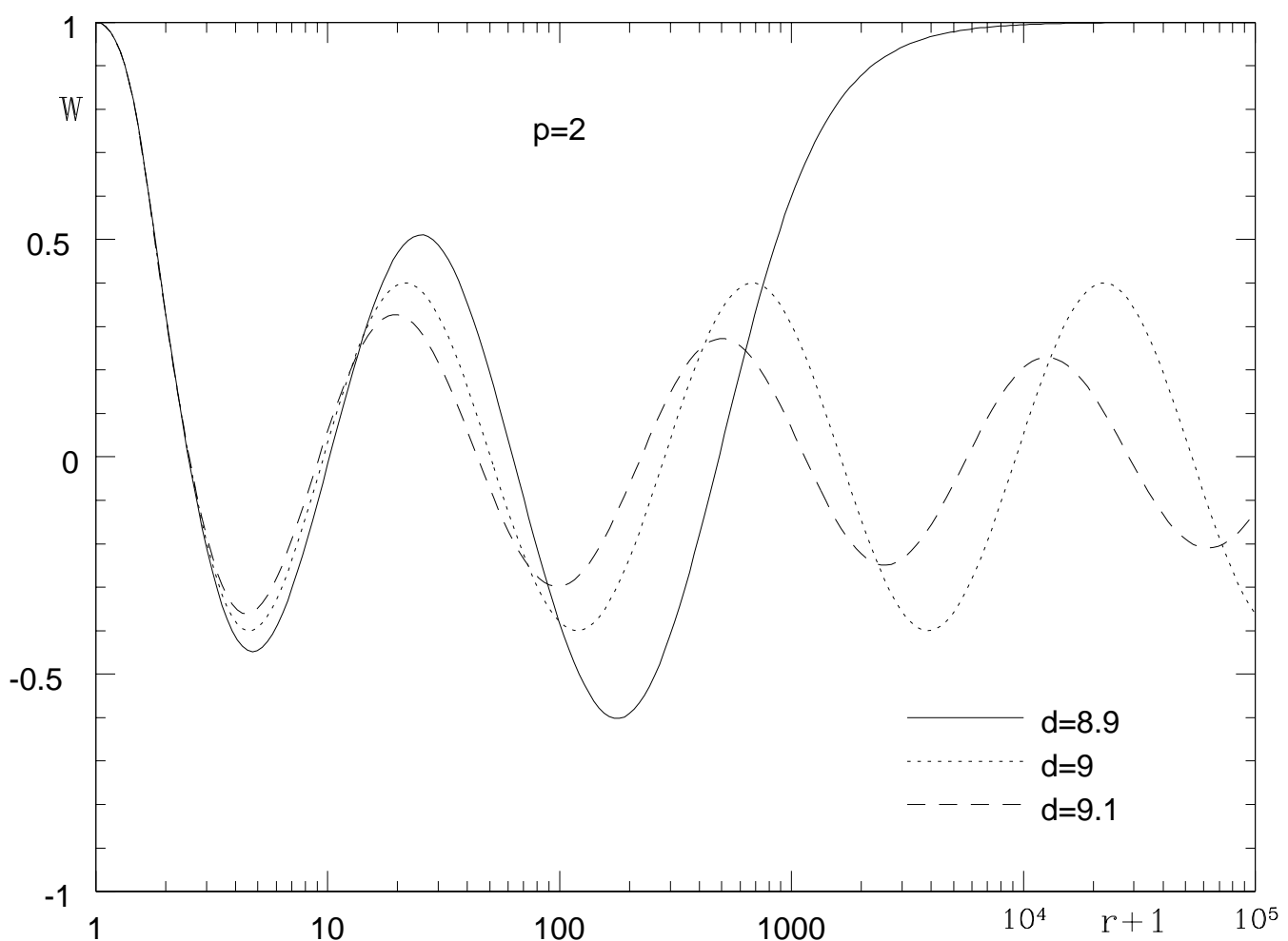

Figure 12: Oscillating solutions of the $p=2$ model for $d=8.9,9$, and 9.1 with decreasing, constant, and growing amplitude respectively 